\title{
Microenvironmental Th9 and Th17 lymphocytes induce metastatic spreading in lung cancer
}

\author{
Ylia Salazar, ${ }^{1}$ Xiang Zheng, ${ }^{1}$ David Brunn, ${ }^{1}$ Hartmann Raifer, ${ }^{2,3}$ Felix Picard, ${ }^{2}$ Yajuan Zhang, ${ }^{2}$ Hauke Winter, ${ }^{4}$ Stefan Guenther, ${ }^{5}$ \\ Andreas Weigert, ${ }^{6}$ Benno Weigmann, ${ }^{7}$ Laure Dumoutier, ${ }^{8}$ Jean-Christophe Renauld, ${ }^{8}$ Ari Waisman, ${ }^{9}$ Anja Schmall, ${ }^{1}$ \\ Amanda Tufman, ${ }^{10}$ Ludger Fink, ${ }^{11}$ Bernhard Brüne, ${ }^{6,12}$ Tobias Bopp, ${ }^{13}$ Friedrich Grimminger, ${ }^{14}$ Werner Seeger, ${ }^{1,14,15}$ \\ Soni Savai Pullamsetti, ${ }^{1,14}$ Magdalena Huber, ${ }^{2}$ and Rajkumar Savai $i^{1,12,14,15}$ \\ 1'Max Planck Institute for Heart and Lung Research, Department of Lung Development and Remodeling, member of the German Center for Lung Research (DZL), member of Cardio-Pulmonary Institute \\ (CPI), Bad Nauheim, Germany. ${ }^{2}$ Institute for Medical Microbiology and ${ }^{3}$ CoreFacility Flow Cytometry, University of Marburg, Marburg, Germany. ${ }^{4}$ Translational Research Unit, Thoraxklinik at Heidelberg \\ University, member of the DZL, Heidelberg, Germany. ${ }^{5}$ Bioinformatics and Deep Sequencing Platform, Max Planck Institute for Heart and Lung Research, Bad Nauheim, Germany. ${ }^{6}$ Institute of Biochemistry I, \\ Faculty of Medicine, Goethe University Frankfurt, Frankfurt, Germany. ${ }^{7}$ Department of Medicine 1, Friedrich-Alexander University Erlangen-Nuremberg, Erlangen, Germany. ${ }^{8} \mathrm{de}$ Duve Institute, Université \\ Catholique de Louvain, Brussels, Belgium. ${ }^{9}$ Institute for Molecular Medicine, University Medical Center of the Johannes Gutenberg University of Mainz, Mainz, Germany. ${ }^{10}$ Respiratory Medicine and Thoracic \\ Oncology, Internal Medicine V, Ludwig-Maximilians-University of Munich and Thoracic Oncology Centre, member of the DZL, Munich, Germany. "Institute of Pathology and Cytology, Wetzlar, Germany. \\ ${ }^{12}$ Frankfurt Cancer Institute, Goethe University, Frankfurt am Main, Germany. ${ }^{13}$ Institute for Immunology, University Medical Center of the Johannes Gutenberg-University, Mainz, Germany. Research Center \\ for Immunotherapy and University Medical Center, Johannes Gutenberg-University, Mainz, Germany. Cerman Cancer Consortium, Heidelberg, Germany. ${ }^{14}$ Department of Internal Medicine, member of the \\ DZL, member of CPI, Justus Liebig University, Giessen, Germany ${ }^{15}$ Institute or Lung Health (ILH), Justus Liebig University, Giessen, Germany.
}

\begin{abstract}
Immune microenvironment plays a critical role in lung cancer control versus progression and metastasis. In this investigation, we explored the effect of tumor-infiltrating lymphocyte subpopulations on lung cancer biology by studying in vitro cocultures, in vivo mouse models, and human lung cancer tissue. Lymphocyte conditioned media (CM) induced epithelial-mesenchymal transition (EMT) and migration in both primary human lung cancer cells and cell lines. Correspondingly, major accumulation of Th9 and Th17 cells was detected in human lung cancer tissue and correlated with poor survival. Coculturing lung cancer cells with Th9/Th17 cells or exposing them to the respective CM induced EMT in cancer cells and modulated the expression profile of genes implicated in EMT and metastasis. These features were reproduced by the signatory cytokines IL-9 and IL-17, with gene regulatory profiles evoked by these cytokines partly overlapping and partly complementary. Coinjection of Th9/Th17 cells with tumor cells in WT, Rag1-/-, II9r/-, and II17 $\mathrm{ra}^{-1-}$ mice altered tumor growth and metastasis. Accordingly, inhibition of IL-9 or IL-17 cytokines by neutralizing antibodies decreased EMT and slowed lung cancer progression and metastasis. In conclusion, Th9 and Th17 lymphocytes induce lung cancer cell EMT, thereby promoting migration and metastatic spreading and offering potentially novel therapeutic strategies.
\end{abstract}

\section{Introduction}

Lung cancer is the leading cause of cancer mortality worldwide, accounting for up to $19 \%$ of all cancer-related deaths. Numbers of deaths due to lung cancer are even greater than the cumulative deaths due to colon, breast, and prostate cancer. Although modern medicine has advanced at great strides, a meager rise in the survival rate in patients suffering from non-small cell lung cancer (NSCLC) advocates a need for further innovative treatment strategies in this disease $(1,2)$.

Accepting the tumor microenvironment as a key player in tumor growth and metastasis enables the identification of nov-

\section{Related Commentary: p. 3409}

Authorship note: SSP, MH, and RS contributed equally to this work. Conflict of interest: The authors have declared that no conflict of interest exists. Copyright: ( 2020 , American Society for Clinical Investigation. Submitted: August 6, 2018; Accepted: March 24, 2020; Published: June 2, 2020. Reference information: / Clin Invest. 2020;130(7):3560-3575. https://doi.org/10.1172/JCl124037. el targets and the development of novel therapies. Tumor-associated stroma consists of, among others, various immune cells, including $\mathrm{CD}^{+}$and $\mathrm{CD} 8^{+} \mathrm{T}$ cells. The immune system, in addition to serving as the first line of defense against microbial pathogens, provides immune surveillance by restricting tumor proliferation, especially via $\mathrm{T}$ cells. However, it has also been shown that under certain circumstances $\mathrm{T}$ cells can aid the tumor to escape immune control and promote proliferation of cancer cells (3). Cytotoxic CD8 ${ }^{+} \mathrm{T}$ cells are known to secrete IFN- $\gamma$ and TNF- $\alpha$, play an important role in the defense against intracellular pathogens and tumor cells (4), and are mostly associated with increased survival of patients in almost all cancers (5). On the contrary, among $\mathrm{CD} 4^{+}$cells (Th cells) are several subpopulations, which may well exert divergent functions, depending on the cytokines secreted in the tumor microenvironment (6, 7). In particular, while Th1 lymphocytes demonstrate an antitumorigenic effect, Th2 cells were suggested as protumorigenic mediators $(8,9)$. Thus, a balance of these protumorigenic or antitumorigenic effects and signaling cascades may effect tumor progression and metastasis. 
Apart from Th1 and Th2 lymphocytes, other subpopulations, in particular Th9 and Th17 lymphocytes, have been implicated in carcinogenesis. Th9 cells are characterized by the expression of IL-9, IL-21, and IL-10 and have been suggested to be antitumorigenic (melanoma) or protumorigenic (lymphoma, lung cancer) (10-12). However, the mechanisms underlying these effects remain largely elusive. Th17 cells are characterized by the production of IL-17A through IL-17F, IL-21, and GM-CSF. Similar to that of Th9 cells, there is considerable debate on the role of Th17 cells in tumor immunity, with evidence for a protective role in melanoma and leukemia versus promotion of tumor growth in colorectal cancer. Thus, the effect of Th9 and Th17 lymphocytes on carcinogenesis may largely depend on the tissue/cancer context (13-15). In the field of lung cancer research, focus has been laid on the Th17/Treg dynamics and their effect on NSCLC biology (16).

Epithelial-mesenchymal transition (EMT) has been reported to be a key phenomenon through which epithelial cancer cells differentiate into highly motile mesenchymal cells, which form the basis of circulating tumor cells. These circulating tumor cells are ultimately responsible for disease progression with metastatic spreading. Although the tumor immune microenvironment has been shown to effect cancer cell EMT through crosstalk with cancer cells (17), the underlying mechanisms are largely unknown.

In this series of human and rodent experiments, we demonstrated that the crosstalk between Th9/Th17 lymphocytes and lung cancer cells may promote tumor progression and, in particular, metastasis. Release of the signature ILs IL-9 and IL-17 by tumor-infiltrating Th9/Th17 lymphocytes was suggested to induce EMT in lung cancer cells, promoting migration and metastatic spreading and suggesting anti-IL-9/IL-17 treatment as strategy in lung cancer.

\section{Results}

Lymphocyte conditioned medium induces EMT and enhances migration of lung cancer cells. In order to dissect the role of lymphocytes and their crosstalk with lung cancer cells, we established a coculture system of either lymphocytes or lymphocytes activated with anti-CD3/CD28 (Figure 1) or PMA (Supplemental Figure 2; supplemental material available online with this article; https://doi. org/10.1172/JCI124037DS1) and a human lung tumor cell line (A549) as well as primary human NSCLC cells (hereafter referred as PTCs; Figure 1 and Supplemental Figure 2). Whereas conditioned medium (CM) from either cell types did not induce EMT, $\mathrm{CM}$ from lymphocyte cocultures was found to possess strong EMT-inducing activity. This effect was more pronounced upon activation of the lymphocytes before coculture. Upon stimulation with this activated coculture CM, both cell types (A549 and PTC) acquired a spindle-like form (mesenchymal phenotype), exhibiting larger gaps between single cells (Figure 1A and Supplemental Figure 2A). At the molecular level, this was accompanied by a reduction in the expression of the epithelial markers (E-cadherin, zonula occludens-2 [ZO2] and cytokeratin 18) and an increased expression of mesenchymal markers (vimentin and $\mathrm{N}$-cadherin) in PTC as well as in A549 cells (Figure 1, B and C; Supplemental Figure 1, A and B; Supplemental Figure 2, B and C; and Supplemental Figure 3, A and B). These results were further confirmed by immunostaining: an apparent loss of cell-to-cell contact and an increase in cytoskeletal filaments and vimentin expression was noted after exposure of the tumor cells to CM from activated lymphocytes or lymphocyte-A549 cocultures (Figure 1D and Supplemental Figure 2D). Furthermore, stimulation of A549 cells with activated coculture CM for 24 hours not only affected epithelial and mesenchymal markers, but also upregulated the expression of corresponding transcription factors, ZEB1 and ZEB2 (Figure 1E and Supplemental Figure 2E). Interestingly, CM (from cocultures) strongly induced proliferation and migration of both A549 and PTCs, providing strong functional relevance to our initial findings (Figure 1, F and G, and Supplemental Figure 2, F and G). Moreover, CM from coculture of lymphocytes activated with either CD3-bound antibody or PMA and A549 cells showed increased levels of the cytokines IL-9 and IL-17 (isotypes IL-17A and IL-17F), as assessed by ELISA (Figure $1 \mathrm{H}$ and Supplemental Figure $2 \mathrm{H}$ ), pointing toward a role of these cytokines in directing the proproliferative and promigratory phenotype of CM-exposed tumor cells.

Greater numbers of Th9 and Th17 cells in patient lung cancer tissue negatively correlate with overall survival. In order to assess the clinical relevance of our in vitro findings, we used a computational imaging technology for the simultaneous evaluation of 7 distinct markers, allowing for spatial analysis of distinct $\mathrm{T}$ cell populations within the same human NSCLC tissue section (18). In these studies, we focused on the following set of markers: T cell surface glycoprotein CD4, cytokeratin; transcription factors, STAT3 and PU.1; cytokines, IL-9 and IL-17 with DAPI as a nuclear stain. The spectrally unmixed images were then analyzed to identify different $\mathrm{T}$ cell phenotypes, based on the aforementioned markers, where Th9 cells were identified by $\mathrm{CD} 4^{+}, \mathrm{PU} .1^{+}$, and IL- $9^{+}$staining and Th17 cells by $\mathrm{CD}_{4}^{+}$, $\mathrm{STAT3}^{+}$, and $\mathrm{IL}^{-17^{+}}$staining (Figure 2A). To visualize the location of Th9 and Th17 cells, phenotyping maps were generated based on the aforementioned markers and informed machine-learning algorithms. Cytokeratin was used to identify epithelial cells in tumor samples and to define tumor and stroma. A total of 66 patient samples of various types of NSCLC comprise these tissue microarrays (TMAs) (Table 1). Quantification was performed for all patients and results are shown as percentages in relation to all cells in a tumor tissue core and as an average per patient. Th cells $\left(\mathrm{CD} 4^{+}\right)$in general, including Th9 and Th17 cells, were located predominantly in the stromal area (Figure 2B). But even though both Th9 and Th17 cells shared this feature, the total amount of Th9 versus Th17 cells varied significantly. In order to assess the association of these subpopulations with overall patient survival, the total percentage of $\mathrm{CD} 4^{+}$cells as well as the Th9 or Th17/CD4 ratios were calculated in these patient samples and related to the outcome of the respective patients. Notably, a high number of CD4 cells and a higher ratio of either Th9/CD4 or Th17/CD4 were significantly associated with decreased survival in patients with NSCLC (Figure 2C). In addition, increased numbers of Th9 and Th17 cells were found in human lung tumor tissues compared with nontumor parts (Figure 2, D and E, and Supplemental Figure 4).

Th9 and Th17 CM induces EMT and migration in mouse lung cancer cells. To further analyze the role of Th9 and Th17 subsets in lung tumor development, mouse Th0, Th9, and Th17 cells were generated $(19,20)$. Briefly, naive $\mathrm{CD} 44^{-} \mathrm{CD} 62 \mathrm{~L}^{+} \mathrm{CD} 4^{+} \mathrm{T}$ cells were isolated from the mouse spleen, followed by treatment with cytokines 
A

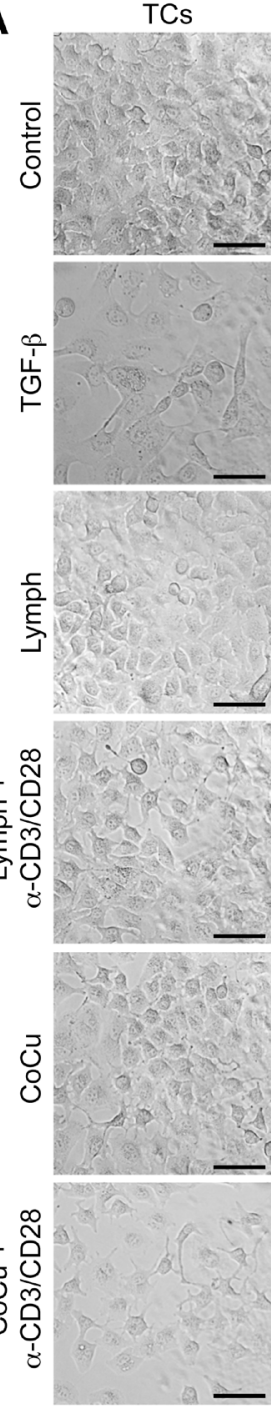

E

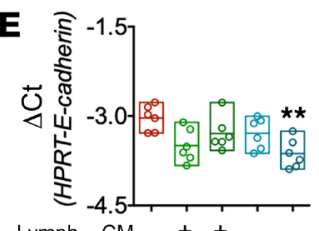

Lymph $-\mathrm{CM}-++--$

$\mathrm{CoCu}-\mathrm{CM}-+-+$

$\alpha-\mathrm{CD} 3 / \mathrm{CD} 28-\ldots++$

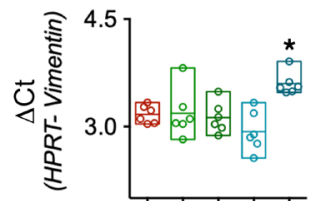

Lymph - CM - + + -

$\mathrm{CoCu}-\mathrm{CM}--++$

$\alpha-\mathrm{CD} 3 / \mathrm{CD} 28-\ldots++$

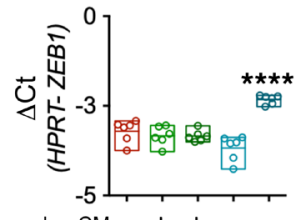

Lymph $-\mathrm{CM}-++-$

$\mathrm{CoCu}-\mathrm{CM}--_{-}^{+}+$

$\alpha-C D 3 / C D 28$
PTCs

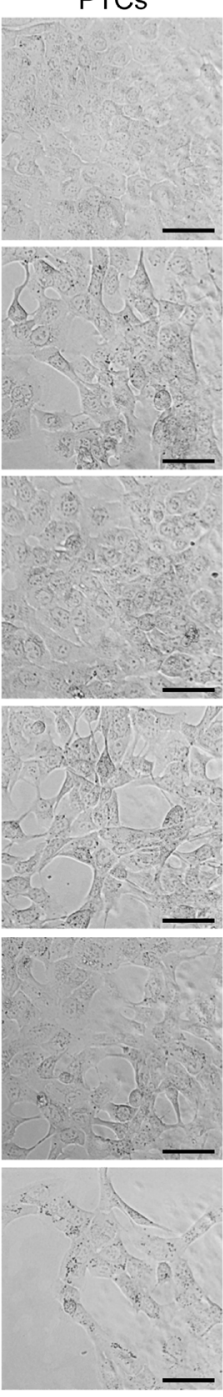

C

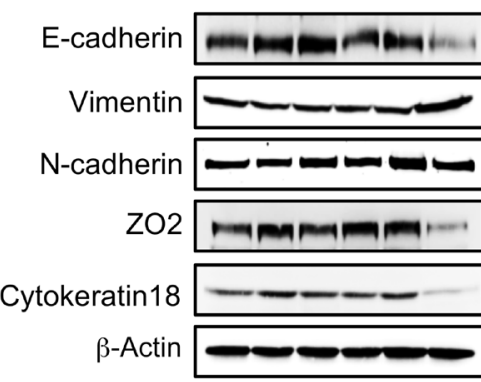

Lymph - CM - - + + - -

$\mathrm{CoCu}-\mathrm{CM}-\quad-\quad++$

$\alpha-\mathrm{CD} 3 / \mathrm{CD} 28---+-+$

TGF- $\beta+----$
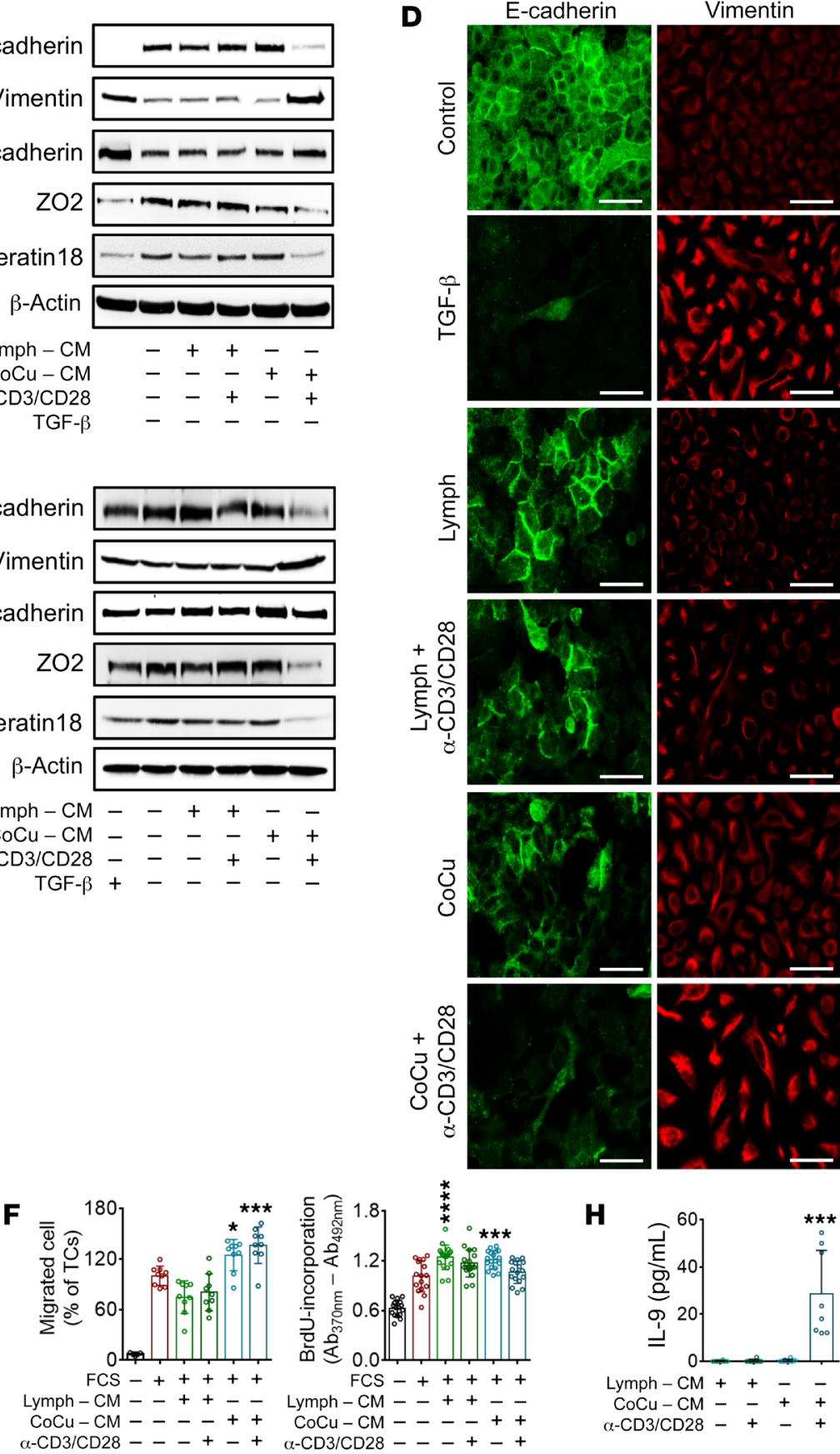

H

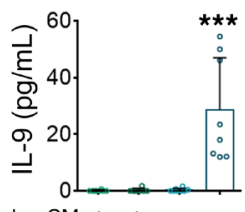

ymph - CM + + - -

$\mathrm{CoCu}-\mathrm{CM}-+++$

$\alpha-\mathrm{CD} 3 / \mathrm{CD} 28-++$

G

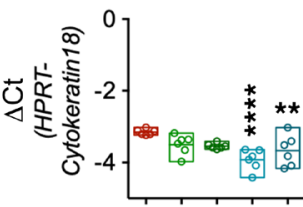

Lymph - CM - + + - -

$\mathrm{CoCu}-\mathrm{CM}-+-++$

$\alpha-C D 3 / C D 28-\ldots++$

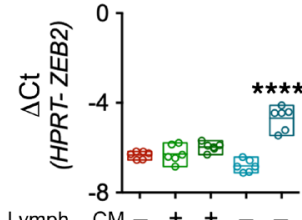

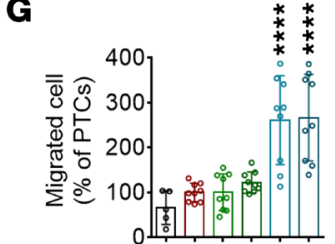

$\mathrm{FCS}-+++++$

Lymph - CM --++--

$\mathrm{CoCu}-\mathrm{CM}---++$

$\mathrm{CoCu}-\mathrm{CM}--+++$ -CD3/CD28 - - + + +
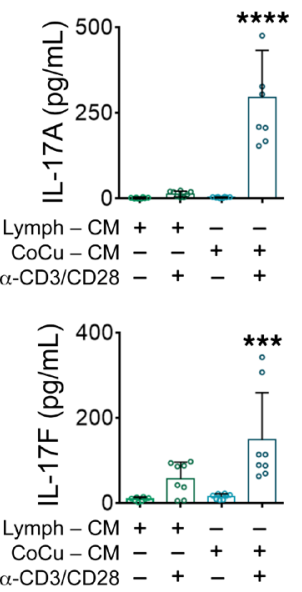
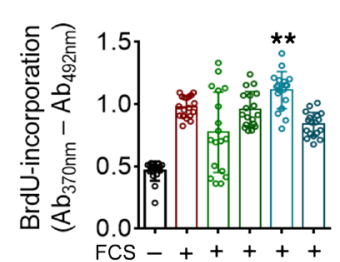

Lymph - CM - - + + -

$\mathrm{CoCu}-\mathrm{CM}---+++$ 
Figure 1. Lymphocyte conditioned medium induces EMT and enhances the migratory potential of human lung cancer cells. Conditioned medium (CM) of A549 cells, primary human NSCLC cells (PTCs), Lymphocytes (Lymph), or coculture (A549/PTCs + Lymph) was used for stimulation of A549 cells and PTCs to assess epithelial-mesenchymal transition (EMT), migration, and proliferation. (A) Representative photomicrographs depicting the morphology of tumor cells (A549 and PTCs) after 48 hours of stimulation with CM. Scale bars: $50 \mu \mathrm{m}$. (B and C) Western blot analysis of EMT markers (E-cadherin, vimentin, $\mathrm{N}$-cadherin, ZO2, and cytokeratin 18) from (B) A549 cells and (C) PTCs lysates after 48 hours stimulation with CM. (D) Immunofluorescence of E-cadherin (green) and vimentin (red) after 48 hours stimulation of A549 cells with CM. Scale bars: $50 \mu \mathrm{m}$. (E) mRNA profile expression of EMT markers after 24 hours stimulation of A549 cells ( $n=3$ donors; 2 experimental replicates). (F) Migration and proliferation (as assessed by BrdU incorporation) of A549 cells after 6 hours and 24 hours of stimulation with CM, respectively. (C) Migration and proliferation (as assessed by BrdU incorporation) of PTCs after 12 hours and 48 hours of stimulation with CM, respectiveIy ( $n=3$ donors). (H) Quantitative analysis of IL-9 and IL-17A detected in CM with or without coculture by ELISA ( $n=4$ donors 2 experimental replicates) ${ }^{*} P<0.05 ;{ }^{*} P<0.01 ;{ }^{* *} P<0.001 ;{ }^{* * *} P<0.0001$ compared with A549, PTCs, or Lymph CM using 1-way ANOVA Dunnett's test.

for 2-3 days to generate Th0 (without skewing cytokines), Th9 (TGF- $\beta$ and IL-4), and Th17 (TGF- $\beta$ and IL-6) cells. Cell differentiation into Th0, Th9, and Th17 types was confirmed by evaluating the corresponding cytokine profile of these cells by FACS (Figure 3A). Subsequently, mouse tumor cell line (LLC1) was either stimulated with the CM of Th0, Th9, or Th17 cell types or cocultured CM for 24 hours (Figure 3B). Interestingly, LLC1 cells stimulated with Th9 or Th17 CM displayed downregulation of $\mathrm{ZO} 2$ and cytokeratin 18 and strong upregulation of fibronectin protein levels (Figure 3C and Supplemental Figure 5A). Th9 CM enhanced proliferation of these tumor cells (Figure 3D) while Th17 CM boosted their migratory potential (Figure 3E). Similarly, CM obtained from coculture of Th0, Th9 or Th17 with LLC1 cells (Figure 3F) demonstrated Th17 influence on EMT marker protein expression and migration (Figure 3, F and H, and Supplemental Figure 5B) while Th9 influenced proliferation of tumor cells (Figure $3 \mathrm{G}$ ).

Coculturing of LLC1 cells with Th9 or Th17 cells alters their gene expression profile. Th9 and Th17 cells were generated as described above and their phenotypes were confirmed through cytokine profile analysis (Supplemental Figure 6A). After 24 hours, cocultured tumor cells were sorted (Supplemental Figure 6B) and analyzed for different gene expression profiles using RNA-Seq. Among all the samples, a high correlation and a clear distinction between conditions were observed by Spearman's correlation (Supplemental Figure 6, C and D). Notably, a total of 3377 genes were found to be differentially regulated $(\mathrm{FDR}<0.05$, base mean $>5$, $\log 2$ fold change $>0.58$ ) in tumor cells cocultured with Th9 cells (LLC1 + Th9) compared with the control group (LLC1 + Th0). Additionally, a total of 500 genes were altered in Th17 cocultured tumor cells (LLC1 + Th17) (Figure 4A). Pathway enrichment analysis in PANTHER database for all differentially expressed genes revealed that Th9 and Th17 coculture groups displayed regulation of common pathways, such as integrin signaling pathway, $\mathrm{T}$ cell activation, and PDGF and EGF receptor signaling. Interestingly, both cell types (Th9 and Th17) induced upregulation of pathways specific for the respective coculture condition. For instance, while the Th9 coculture group (LLC1 + Th9) showed an increase in the chemokine-mediated inflammation and angiogenesis-related pathway, the Th17 coculture group (LLC1 + Th17) exhibited an increase in the oxidative stress response and Ras signaling pathway (Figure 4B). Furthermore, Figure 4, C and D, respectively, depict protein-coding genes (Figure $4 \mathrm{C}$ ) and transcription factors (Figure 4D) strongly regulated upon LLC1 coculture with Th9 and Th17 cells. Notably, the LLC1 + Th9 group showed regulation of genes involved in cell growth, proliferation, and metastasis, such as MMP3, MMP13, PlexinA4, complement component 3 (C3), and Cxcl1. In the case of the LLC1 + Th17 group, upregulated genes included Ywhaz, Psat1, and $L y 6 e$, which are known to be involved in various key aspects of the metastatic phenotype, including promotion of EMT, cell cycle progression, and regulation of tumor vessel density (Figure 4C). Interestingly, common transcription factors were upregulated in the LLC1 + Th9 and LLC1 + Th17 groups, including Krüppel-like factors (Klfs). Among these, Klf4 and Klf5 are regulated in the LLC1 + Th9 group, whereas Klf7 is regulated in the LLC1 + Th17 group. In addition, known tumor suppressor genes, such as Notch1 and Hic1, were found to be downregulated in the LLC1 + Th9 group (Figure 4D).

Coinjection of tumor cells and Th9/Th17 cells promotes lung cancer growth and particularly metastasis in the mouse model. Mouse Th9 and Th17 cells were generated and their phenotype was confirmed through cytokine profile analysis (Supplemental Figure 7, A and B). These cells were then coinjected intravenously with LLC1 cancer cells into immunodeficient $\mathrm{Rag1}^{-/-}$mice, and lung metastasis was assessed at day 20 with the use of Micro CT imaging (Figure 5A). Coinjection of tumor cells with Th9 and Th17 cells significantly enhanced metastasis in $\mathrm{Rag1}^{-/-}$mice, while administration of neutralizing antibodies against cytokines IL-9 and IL-17 successfully counteracted this effect (Figure 5, B and C).

Immunofluorescence staining of lung sections for the EMT marker vimentin confirmed an upregulation in mice coinjected with Th9 and Th17 cells (Figure 5B and Supplemental Figure 7E). As expected, $\mathrm{CD}^{+}$cells were increased solely in lungs of $\mathrm{Rag1}^{-/-}$ mice coinjected with $\mathrm{T}$ cells, as assessed by flow cytometry (Figure 5, D-F). Other immune cells (monocytes, B cells, and CD8 ${ }^{+} \mathrm{T}$ cells) remained unchanged (Figure 5, E and F, and Supplemental Figure 7, C and D).

Table 1. Patient information included in the present study

\begin{tabular}{lc} 
Variable & 66 \\
Number & $65(38-83)$ \\
\hline Median age in years at operation (range) & $3(0.7-13)$ \\
\hline Median tumor size (range), CM & $49(1-110)$ \\
\hline Median overall survival in months (range) & 61 \\
\hline Reached cancer survival endpoint & \\
Sex & 40 \\
\hline Male & 26 \\
\hline Female & 32 \\
Subtype & 26 \\
\hline Adenocarcinoma & 6 \\
\hline Squamous cell carcinoma & 2 \\
\hline Large cell carcinoma & \\
\hline Unidentified &
\end{tabular}



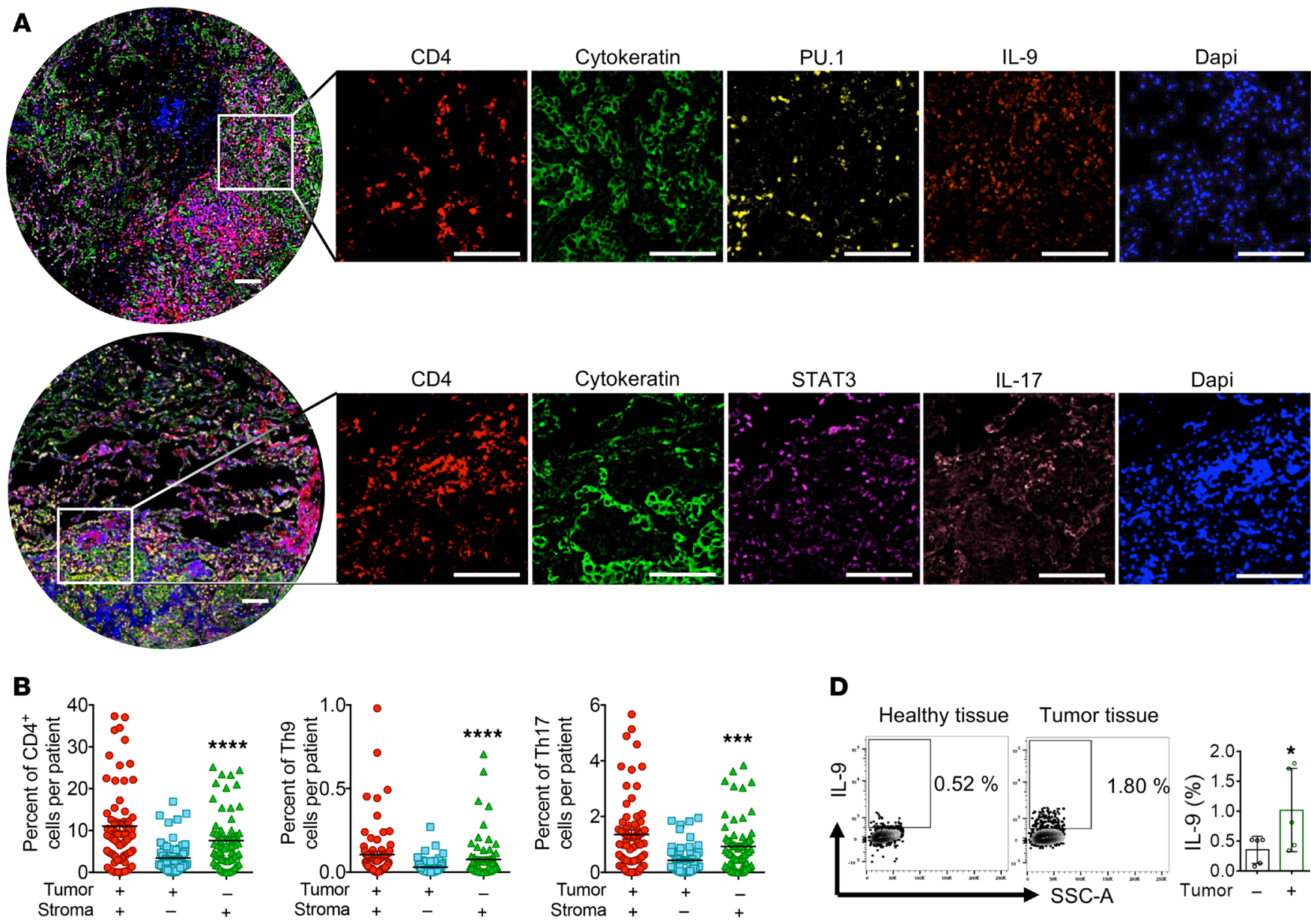

D
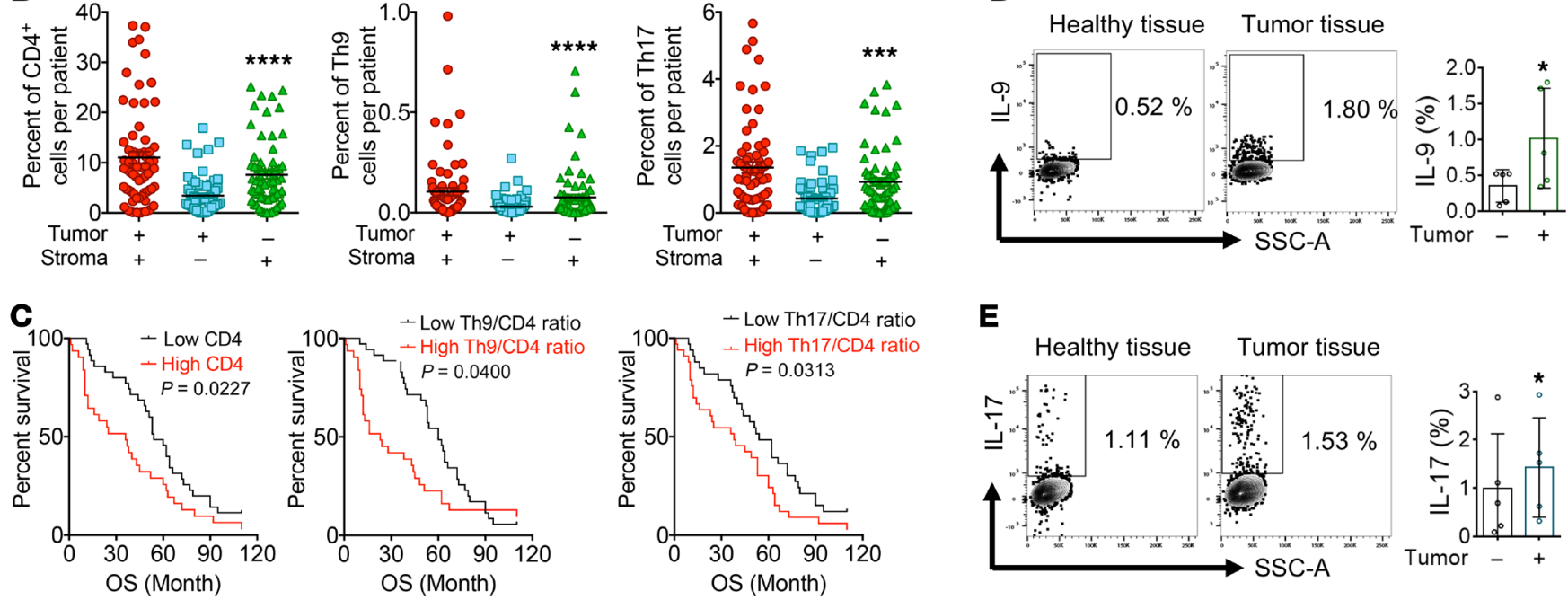

Figure 2. Analysis of Th9 and Th17 cells in human lung cancer by Opal multiplexed staining. (A) Representative multiplex immunofluorescence images of NSCLC specimens (adenocarcinoma, $n=32$; squamous cell carcinoma, $n=26$; large-cell carcinoma, $n=6$; unidentified, $n=2$; details given in Table 1) displaying 2 tissue microarrays (TMA) cores after multispectral imaging and enlarged subsections of the core showing each of the individual markers in the composite image after spectral unmixing. Markers: CD4 (Opal 620, pseudocolored red), cytokeratin (Opal 520 pseudocolored green), PU.1 (nuclear, Opal 540, pseudocolored yellow), STAT3 (Opal 690, pseudocolored magenta), IL-9 (Opal 570, pseudocolored orange), IL-17 (Opal 650, pseudocolored pink), and DAPI as a nuclear marker (pseudocolored blue). Scale bars: $100 \mu \mathrm{m}$. (B) Percentage of CD4+, Th9, and Th17 cells in tumor and stroma of all patients with $\operatorname{NSCLC}(n=66) .{ }^{* *} P<0.001 ;{ }^{* * *} P<0.0001$ when comparing percentage of cells between tumor and stroma using unpaired $t$ test. (C) Survival analysis of 66 patients with NSCLC based on the Th9/CD4+ and Th17/CD4+ ratios. $P$ values reflect the comparison between high and low ratio groups by using log-rank test. High- and low-ratio group values were defined based on the median percentage of positive cells or ratio. (D) FACS analysis of Th9 cells in human lung tumor tissue $(n=5)$. (E) FACS analysis of Th17 cells in human lung tumor tissue $(n=5)$. ${ }^{*} P<0.05$ when comparing to normal lung tissue using paired $t$ test.

In addition, coinjection of LLC1 and Th9 cells significantly enhanced subcutaneous tumor growth and weight in WT mice, when compared with control group (Supplemental Figure 8B). Surprisingly, Th17-LLC1 coinjection resulted in a greater incidence of metastatic nodules without affecting tumor size (Supplemental Figure 8, B-E). Immunofluorescence of tumors displayed an increase of vimentin in both the Th9 and Th17 coin- jection groups (Supplemental Figure 8D). To further understand the role of these immune cells in metastasis, we coinjected Th9 and Th17 cells with LLC1 cells intravenously in WT mice. Interestingly, greater number of lung tumors were observed in mice coinjected with Th9 and Th17 cells (Supplemental Figure 8, G and F). In line with the observation in subcutaneous tumors, lung tumor immunofluorescence showed an increase in the mesen- 

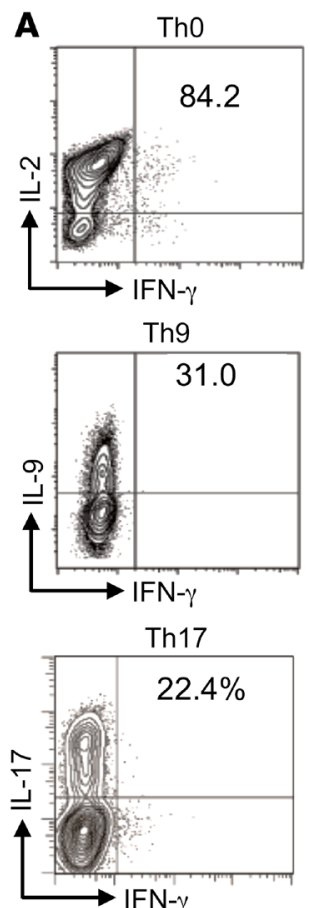

B Th0 or Th9 or Th17
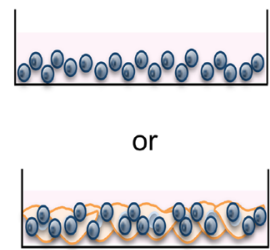

TCs + Th cells

Cocultures

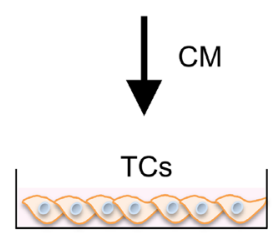

Proliferation

Migration

EMT
C

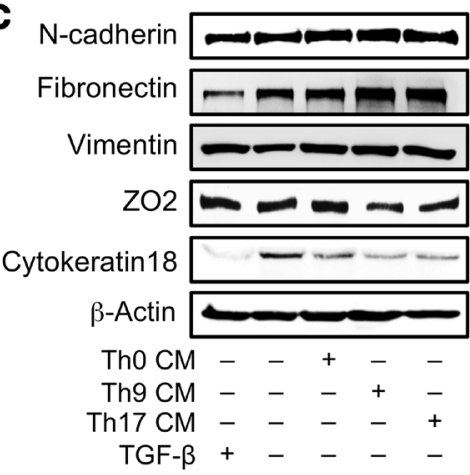

E

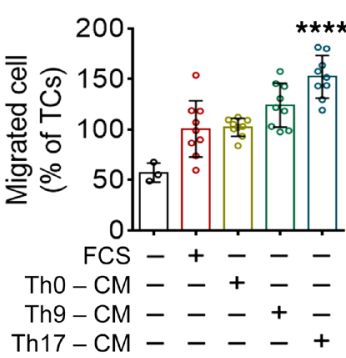

D

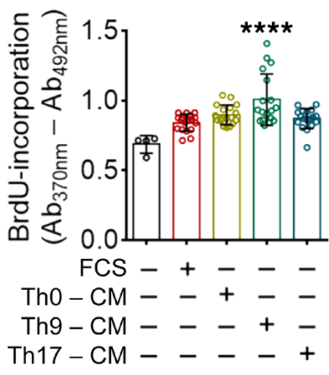

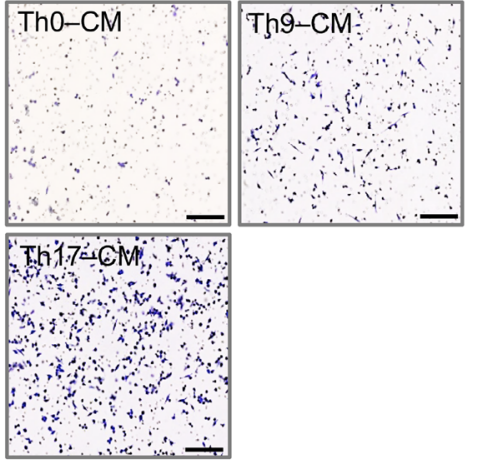

F

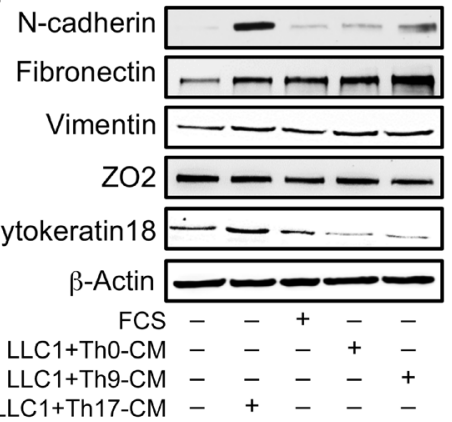

G

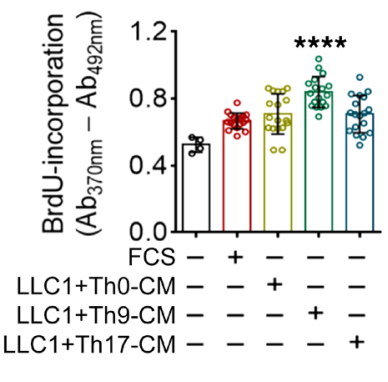

H

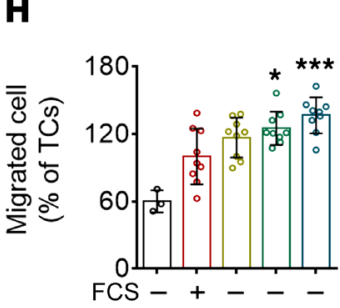

LLC1+ThO-CM - + + - -

LLC1+Th9-CM - - - +

LLC1+Th17-CM - - - +

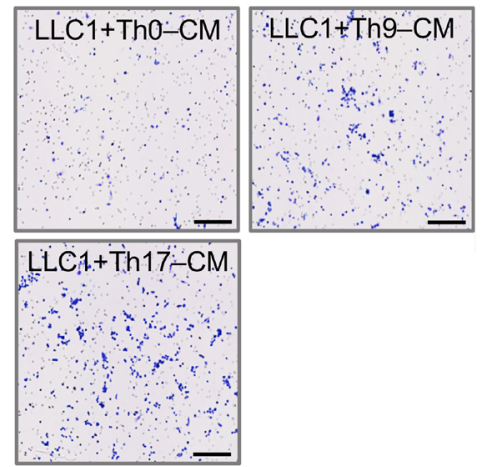

Figure 3. $\mathrm{CD}^{+} \mathrm{T}$ cell subtype-specific $\mathrm{CM}$ induces EMT and migration in mouse cancer cells. (A) Naive CD44-CD62L $\mathrm{L}^{+} \mathrm{CD} 4^{+} \mathrm{T}$ cells were isolated from C57BL/6 mice and treated with specific cytokines for 2-days to differentiate into Th9 (TCF- $\beta$ and IL-4), Th17 (TGF- $\beta$ and IL-6), or Th0 (without skewing cytokines) cells. Differentiation was confirmed by performing analysis for production of cell-specific cytokines, such as IL-2, IL-9, IL-17, and IFN- $\gamma$ by flow cytometry. (B) Schematic experimental design showing CM from Th0, Th9, or Th17 cells or from their coculture with cancer cells used to stimulate LLC1 cells and evaluate EMT, proliferation, and migration. (C) Western blot analysis of EMT markers (N-cadherin, fibronectin, vimentin, ZO2, and cytokeratin 18) from LLC1 cell lysate after 48 hours of stimulation with Th0, Th9, or Th17 CM. (D and E) Proliferation and migration of LLC1 cells after 12-hour and 24-hour stimulation, respectively, with CM. (F) Western blot analysis of EMT markers (N-cadherin, fibronectin, vimentin, ZO2, and cytokeratin 18) from LLC1 cell lysate after 48-hour stimulation with respective coculture CMs. (G and $\mathbf{H})$ Migration and proliferation of LLC1 cells after 12-hour and 24-hour stimulation, respectively, with coculture CMs. ( $n=3$ donors) ${ }^{*} P<0.05$; ${ }^{* *} P<0.001 ;{ }^{* * *} P<0.0001$ compared with LLC1 using 1-way ANOVA Dunnett's test. Scale bars: $500 \mu \mathrm{m}$.

chymal marker vimentin in the Th9 and Th17 coinjection groups (Supplemental Figure 8F).

IL-9 and IL-17 induce EMT in human tumor cells. To further delineate the effects of Th9 and Th17 cells on tumor cells in vitro, A549 cells were stimulated with their corresponding signatory cytokines, IL-9 and IL-17 (IL-17A and IL-17F), for 48 hours. IL-9 induced EMT in A549 cells, as observed by the changes in protein expression of E-cadherin and vimentin, and were accompanied by enhanced migration (Figure 6, A-C, and Supplemental Figure 9A). A similar effect was observed for IL-17A and IL-17F (Figure 6, D-I, and Supplemental Figure 9, B and C). However, IL-17A regulated EMT and migration more prominently in lower concentration ranges compared with IL-17F. Corroborating this finding, IL-9, IL-17A, and IL-17F also enhanced migration in PTCs (Figure 6J).

Coinjection of LLC1 cells with Th9 or Th17 in IL-17R-and IL-9Rdeficient mice leads to enhancement of metastasis in discordant transfer setting. Th9 and Th17 cells were coinjected intravenously with LLC1 cancer cells into Il17 $\mathrm{ra}^{-1}$ and $\mathrm{Il9r} /$ mice, and lung metastasis was assessed at day 20 (Figure 7, A and H). Using these transgenic mice, we found that coinjection of LLC1 cells with Th9 or Th17 in 
A MA plot, LLC1+Th9
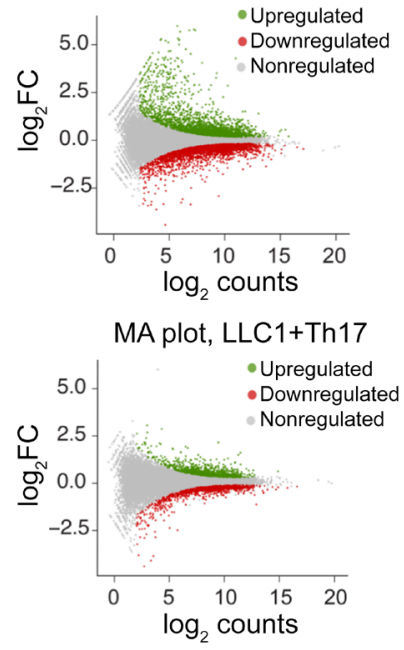

C

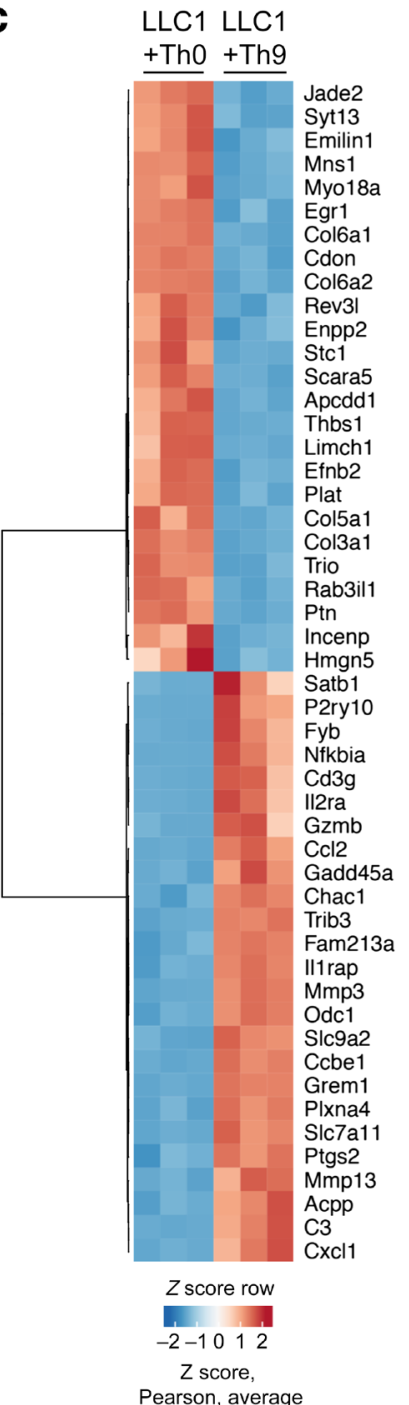

B
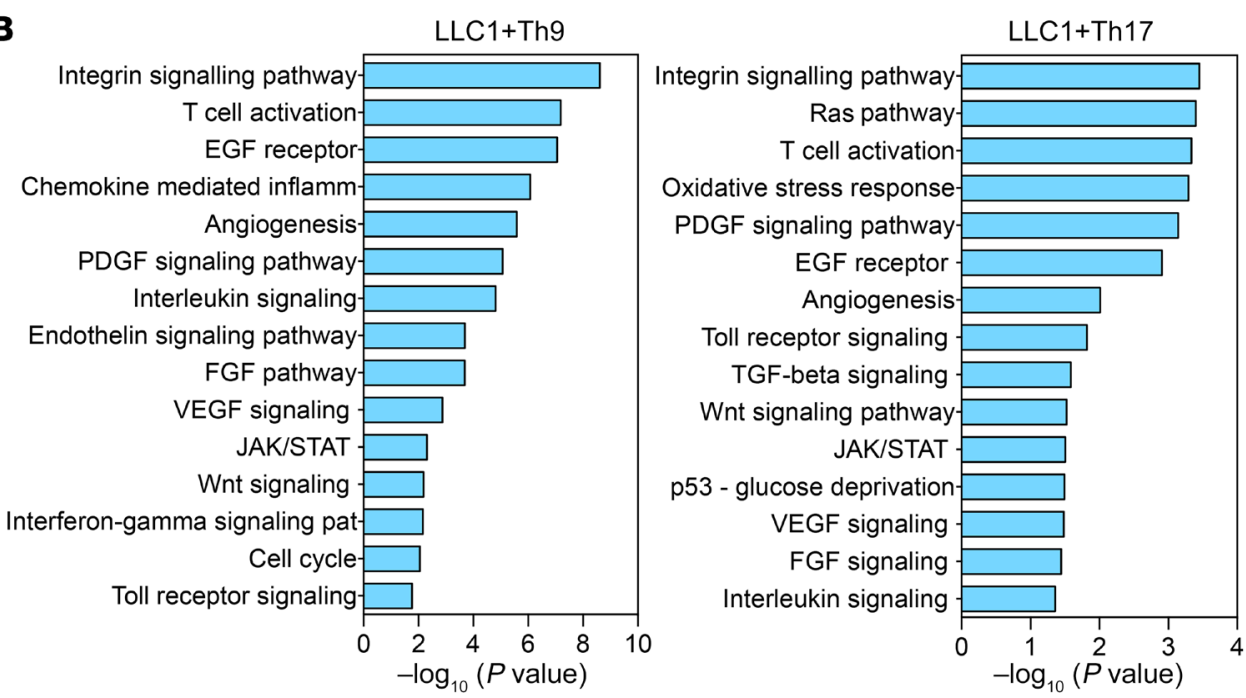

LLC1 LLC1
+ Th0 +Th17
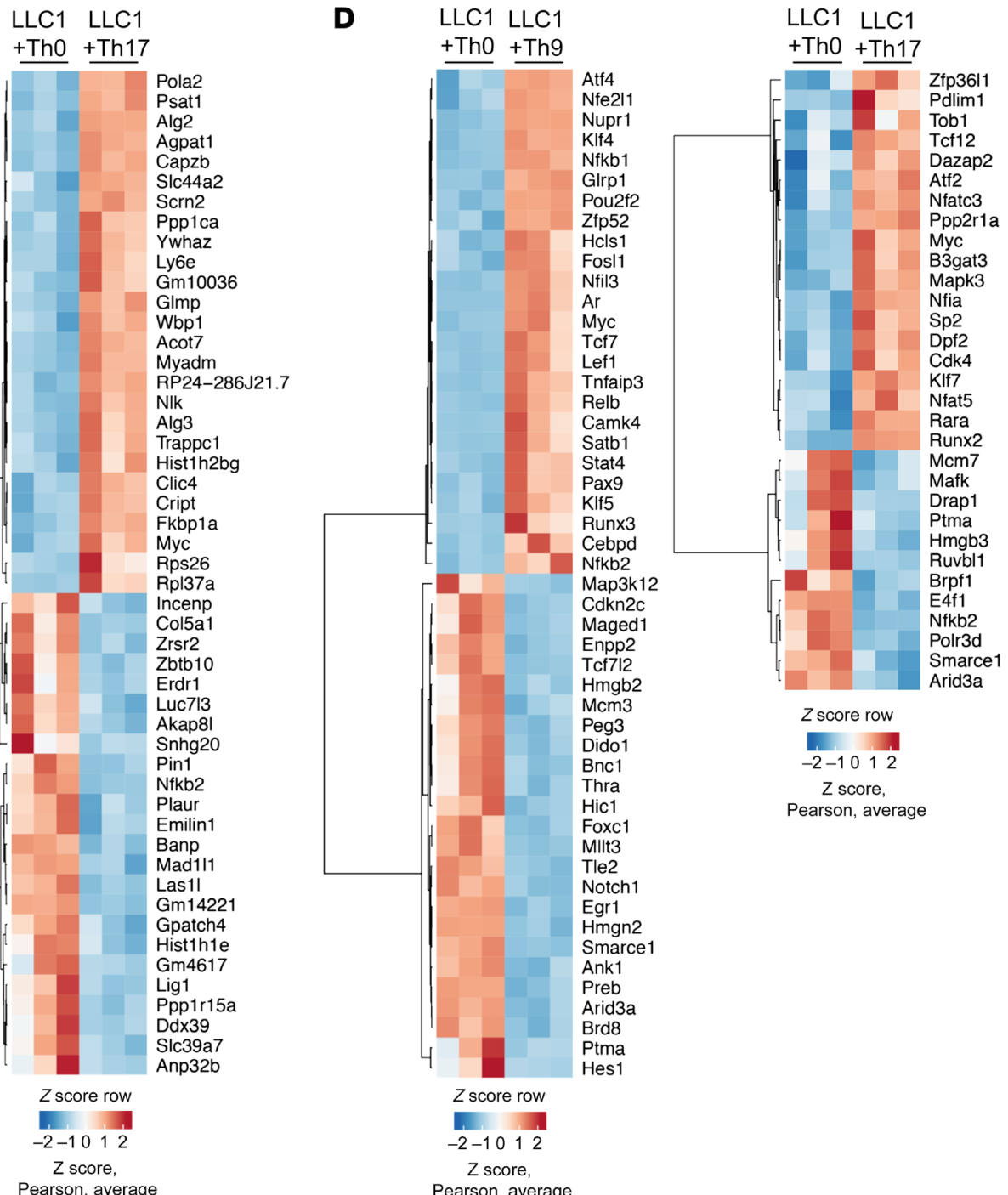

Pearson, average 
Figure 4. Coculture with Th9 or Th17 cells affects tumor cell gene profile. (A) MA plot of RNA-Seq data showing $\log _{2}$ average signal intensity of treatment groups ( $y$ axis) versus $\log _{2}$ average signal intensity of control group (LLC1 + ThO; $x$ axis). Regulated genes are colored based on adjusted $P$ value (FDR) $<0.05$ (B) Pathway enrichment analysis in PANTHER database for differentially regulated genes in LLC1 cells cocultured with Th9 or Th17 cells as compared with ThO. (C) Heatmap of differentially expressed genes ( $Z$ score normalized) in LLC1 + Th9 and LLC1 + Th17 groups. Genes with higher expression levels are shown in red, while genes with lower expression levels are shown in blue. (D) Heatmap of differentially expressed transcription factors in LLC1 + Th9 and LLC1 + Th17 groups. $Z$ score normalized gene expression is shown for top25 upregulated and downregulated genes based on lowest adjusted $P$ value. Genes with higher expression levels are shown in red, while genes with lower expression levels are shown in blue. $n=3$ experimental replicates, clustering of heatmaps by Pearson correlation.

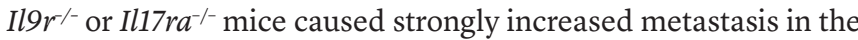
discordant ligand receptor constellation (Th9 in $\mathrm{Il17ra}^{-/-}$mice and Th17 in Il9r/- mice), with only limited efficacy in the concordant constellation (Th9 in $\mathrm{Il}_{19 \mathrm{ra}^{--}}$mice and Th17 in $\mathrm{Il7ra}^{-/-}$mice) (Figure 7, B, C, I, and J). Likewise, expression levels of the EMT marker vimentin, assessed by immunofluorescence staining, were strongly enhanced in the discordant ligand receptor constellation (Th9

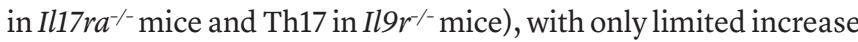
in the concordant constellation (Th9 in $\mathrm{Il19r}^{--}$mice and Th17 in $\mathrm{Il7ra}^{-/-}$mice) (Figure 7, B and I, and Supplemental Figure 10). Other immune cells remained unchanged (Figure 7, D-G and K-N).

$I L-9$ and IL-17 neutralization decreases lung cancer metastasis. Tumor-bearing mice were treated with specific antibodies against IL-9 and IL-17 (Figure 8A). Notably, anti-IL-9- and anti-IL-17treated groups showed significantly reduced metastatic tumor nodules in comparison with nontreated mice. Combination treatment did not show a synergistic or additive effect on tumor growth inhibition (Figure 8, B and C). In addition, the mesenchymal marker vimentin was markedly decreased in mice treated with IL-9 and IL-17 antibodies in comparison with that in the nontreated group (Figure 8B and Supplemental Figure 11B). Treatment with neutralizing antibodies had no effect on immune cell subpopulations (Figure 8, D and E, and Supplemental Figure 11A).

\section{Discussion}

In this series of human and rodent experiments, we demonstrate that the crosstalk between tumor immune cells and lung cancer cells may promote tumor progression and in particular metastasis. As graphically summarized in Figure 8F, release of the signature cytokines IL-9 and IL-17 by tumor-infiltrating Th9/Th17 lymphocytes is suggested to induce a series of gene-regulatory events in lung cancer cells, changing their profile toward EMT and thereby promoting tumor cell migration, vessel entrance, and metastasis. As shown from our demonstration of these features in vitro, lung cancer cell EMT and metastasis were enhanced by fostering Th9 and Th17 lymphocyte accumulation in the tumor microenvironment and inhibited by antiIL-9 and anti-IL-17 treatment in mouse cancer models in vivo. Correspondingly, enhanced numbers of Th9 and Th17 cells in patient lung cancer tissue were found to be negatively correlated with survival.

EMT is characterized by major phenotypical alterations, such as cytoskeletal reorganization, detachment from the extracellular matrix, and loss of polarity. Several studies have emphasized that EMT drives metastasis during progression of cancer and that certain EMT-inducing signals can also trigger immunosuppressive mechanisms that aid in accelerating the onset of cancer metastasis $(17,21)$. Coculturing of lymphocytes and lung cancer cells in this study was used to mimic their microenvironmental interplay. Analyzing the CM of these cocultures, we consistently observed high levels of IL-9 and IL-17 (isotypes A and F), in particular upon preactivation of the lymphocytes with PMA or anti-CD3/CD28. Prompted by this observation, we analyzed human lung cancer tissue samples and observed high numbers of IL-9- and IL-17-producing $\mathrm{CD}^{+} \mathrm{T}$ cells in the tumor microenvironment. Most importantly, the enhanced appearance of IL-9- and IL-17-producing $\mathrm{CD}^{+} \mathrm{T}$ cells significantly correlated with decreased patient survival. Our results are in agreement with those from Chen et al., who also noted a positive correlation of IL-17-producing cells with decrease in survival in patients with lung cancer (22). In that study, the appearance of intratumoral IL $-17^{+}$cells was found to be correlated with lymphatic vessel density, fostering the proposal that Th17 cells promote tumor development through lymphangiogenesis. However, in other cancers, including leukemia and thyroid cancer, there is considerable controversy on the correlation between the presence of lymphocyte subsets and survival patterns of patients $(23,24)$. In hepatocellular carcinoma, patients with elevated serum levels of IL-9 and higher numbers of circulating and tumor infiltrating Th9 cells presented shorter disease-free survival periods after resection (25).

Focusing on a putative lung cancer-promoting role of IL-9and IL-17-producing CD4 $4^{+} \mathrm{T}$ cells in more detail, we identified upregulation of lung cancer cell EMT as a consistent feature both upon directly coculturing of tumor cells with either Th9 or Th17 or upon exposure of primary human lung cancer cells or cell lines to the respective signatory cytokines IL-9 and IL-17: several EMT markers were upregulated and linked with enhanced cancer cell migration. Correspondingly, RNA-Seq revealed enhanced expression of several MMPs known to play a role in metastatic tumor spreading (26, 27). For instance, Th9 cells strongly regulated MMP3 and MMP13 expression in lung cancer cells, accompanied by an upregulation of other EMT mediators, such as C3, a reported E-cadherin inhibitor (28), and plexin A4, a gene implicated in cytoskeletal reorganization, which was earlier reported to be associated with tumor progression and angiogenesis in melanoma (29). The list of Th9-affected genes also includes the Ywhaz gene, which was reported to influence cell adhesion and motility in several cancers, including NSCLC (30-33), where its expression correlated with stage and grading of the cancer and was inversely related to patient survival (32). A prometastatic function of Ywhaz was previously suggested to be due to its prevention of $\beta$-catenin ubiquitination, with the subsequent nuclear accumulation of $\beta$-catenin, leading to the activation of EMT markers (33). Klfs, which have been earlier identified to be associated with metastasis in numerous cancers and are well established as EMT inducers $(34,35)$, were further discernible markers found to be upregulated in lung cancer cells upon coculture with either Th9 or Th17 cells in the present study. It is of interest to note that despite similar effects of these $2 \mathrm{~T}$ cell subpopulations or their signature cytokines on lung cancer cells, Th9 and Th17 cocultures elicited distinct molecular pathways: whereas 
A

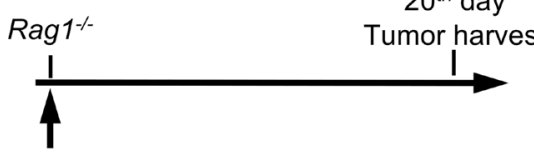

LLC1, Th9 and Th17 cell co-injection (i.v.)
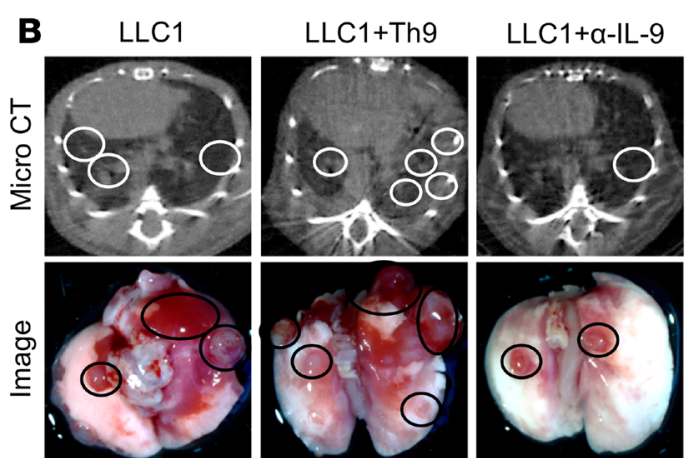

LLC1+
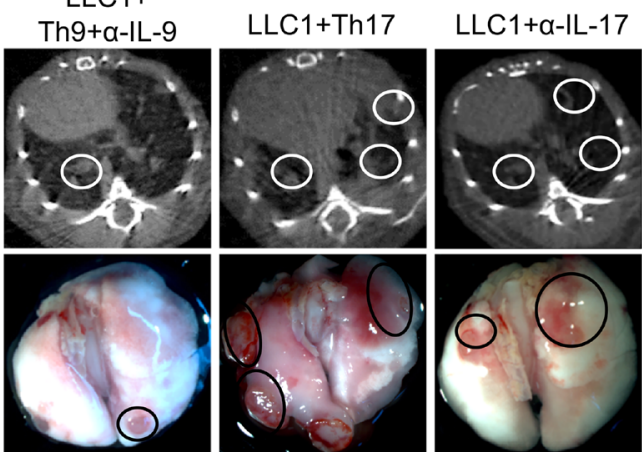

LLC1+
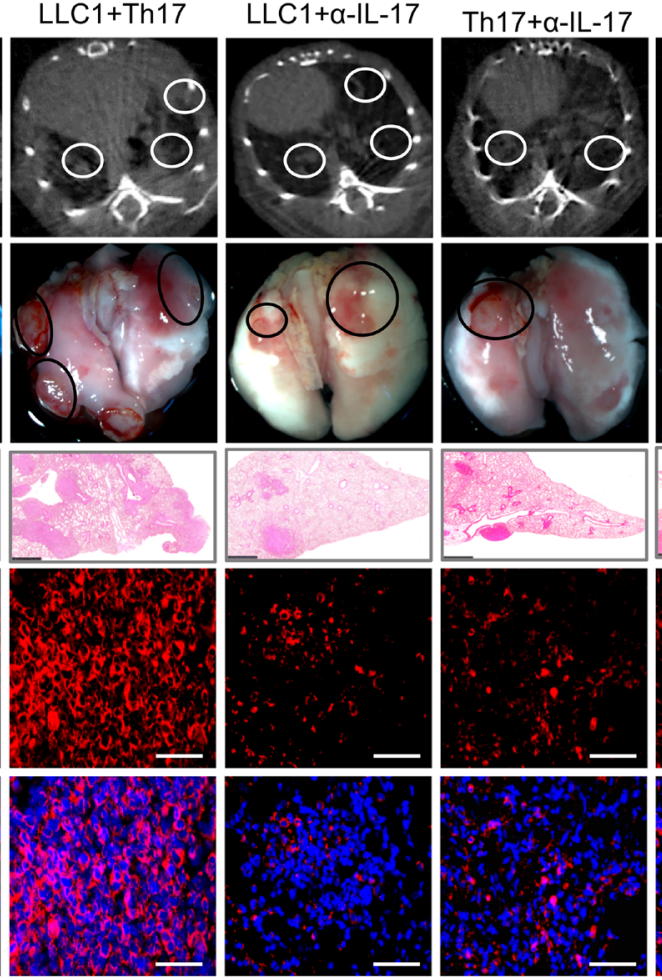

LLC1+

D
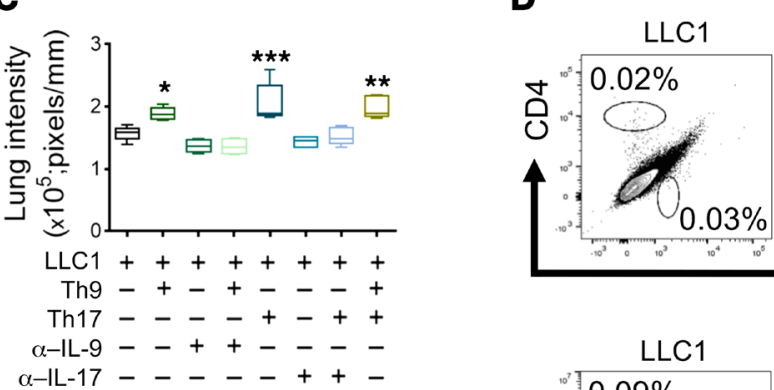

LLC1+Th9

Th9+a-IL-9

LLC1+
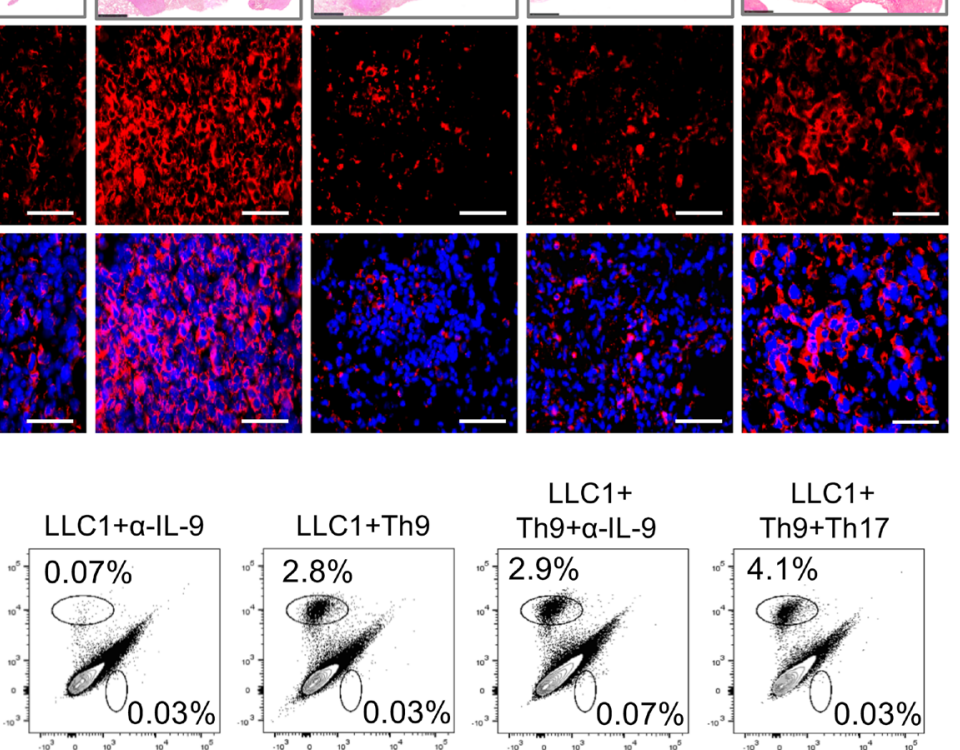

Th9+Th17

LLC1+
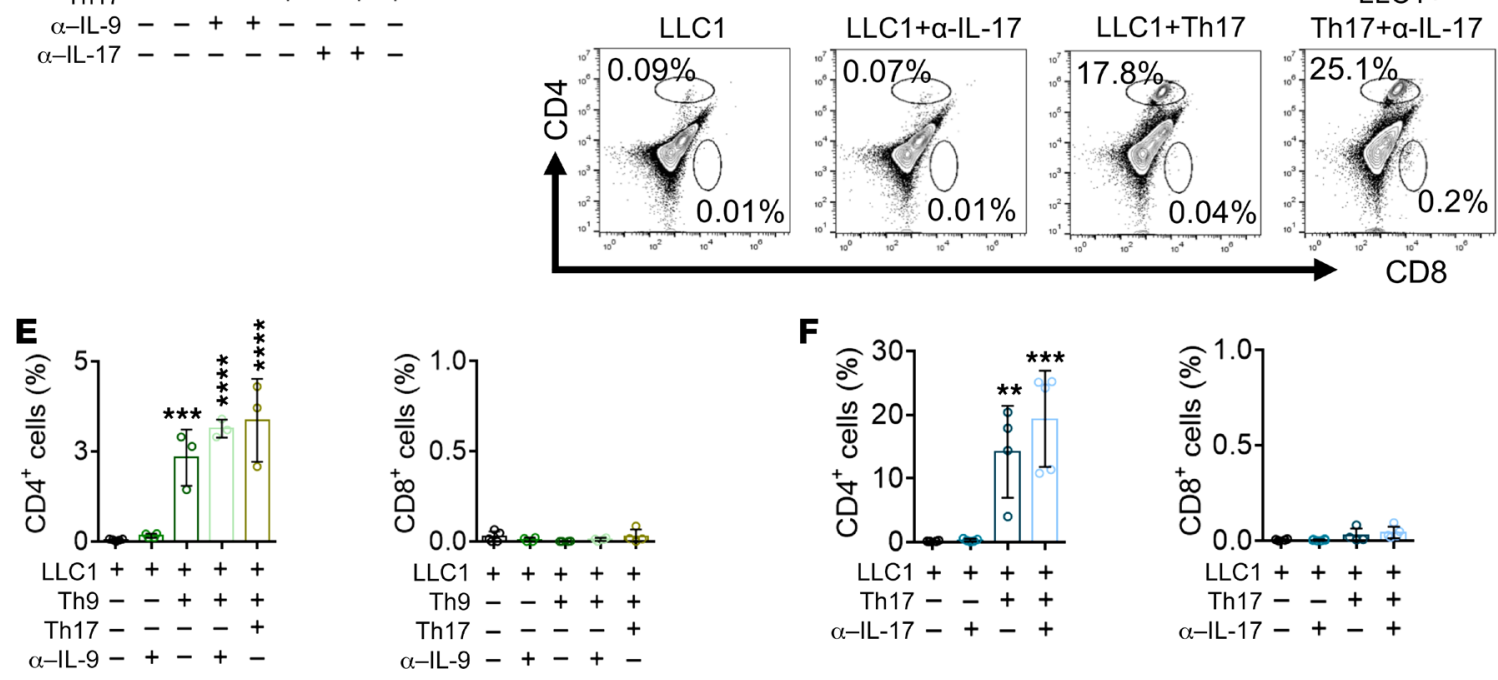

F
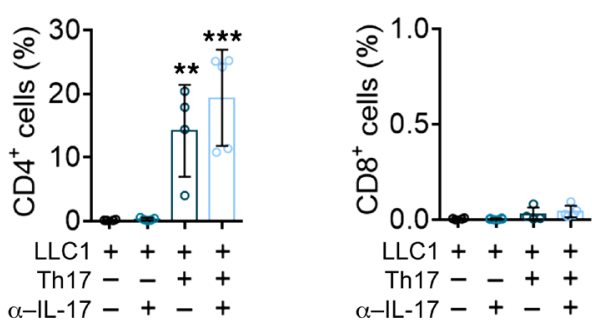
Figure 5. Coinjection of LLC1 cells with Th9 and Th17 cells in immunodeficient mice leads to increased primary tumor growth and metastasis in vivo. (A) Schematic of experimental design. Naive CD44-CD62L+CD4+

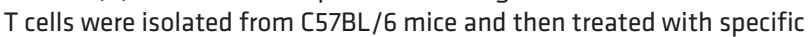
cytokines for 2-3 days in order to differentiate into Th9 (TCF- $\beta$ and IL-4), Th17 (TCF- $\beta$ and IL-6), or Th0 (without cytokines) cells. Differentiation was confirmed by performing analysis for IL-9, IL-17, IL-2, and IFN- $\gamma$ production by flow cytometry. Afterward, Th0, Th9, or Th17 cells were coinjected with LLC1 cells into Rag $^{1 /-}$ mice intravenously followed by anti-IL-9/anti-IL-17 treatment as indicated. (B) Representative images of micro-CT, extracted lungs, and $\mathrm{H} \& \mathrm{E}$-stained sections (scale bars: $1 \mathrm{~mm}$ ), and immunofluorescence staining for vimentin (red) and DAPI (blue) in tumor sections (scale bars: $50 \mu \mathrm{m})$. (C) Quantification of average lung intensity $(n=5)$. (D-F) FACS analysis of CD4+ and CD8 ${ }^{+}$immune cells in lungs from Rag 1/- mice $^{-1}$ after Th9 and Th17 coinjection $(n=3) .{ }^{*} P<0.05 ;{ }^{* *} P<0.01 ;{ }^{* *} P<0.001$; ${ }^{* * *} P<0.0001$ compared with LLC1 using 1-way ANOVA Dunnett's test.

the Th9 "profile" was predominantly characterized by an increase in chemokine-mediated inflammation and angiogenesis, the Th17 cells influenced the oxidative stress response and Ras signaling pathways, thus pointing toward a discrete and putatively complementary molecular role of these cell types in modulating tumor progression and in particular metastasis.

Notably, our in vivo studies with Th9 and/or Th17 coinjection in WT and immunodeficient mouse lung cancer models fully corroborated the in vitro findings with respect to lung cancer EMT induction, tumor growth, and metastatic spreading. In line with the regulation of angiogenic markers observed in vitro upon coculturing, the animal experiments confirmed the modulation of several angiogenic markers, including CD31 and von Willebrand factor, upon coinjection of Th9 and Th17 cells to enhance their presence in the tumor microenvironment. Increased lung metastasis after coinjection of Th9 and Th17 cells in Rag1-/- mice was prevented with the use of neutralizing antibodies, further reinforcing a specific metastatic-promoting role for effector cytokines IL-9 and IL-7. Furthermore, upregulation of MMP9, an important player in metastasis (36), in the tumor stroma of Th17-coinjected mice suggests a role of this metalloproteinase also in Th17-driven lung cancer metastasis. Moreover, the discrete effect observed with respect to increased tumor growth and weight in Th9-coinjected mice and the increase in metastatic nodules without affecting the tumor size in Th17-coinjected mice highlights a distinct but complementary effect of these cell types in promoting an overall effect on tumor progression and metastasis.

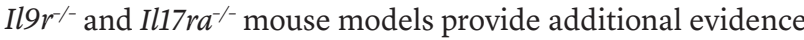
and help elucidate the role of Th9 and Th17 cells and the specificity of their effector cytokines in lung cancer progression. Using these transgenic mice, we found that coinjection of LLC1 cells

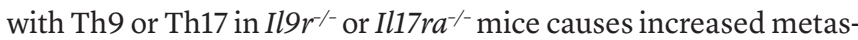
tasis and EMT in the discordant ligand receptor constellation (Th9 in Il17 $\mathrm{ra}^{-1-}$ mice and Th17 in Il9 $\mathrm{ra}^{-1-}$ mice), with only limited efficacy in the concordant constellation (Th9 in Il19r/- mice and Th17 in $\mathrm{Il}_{\mathrm{ra}}^{-1-}$ mice). The remaining activity may be attributed to other cytokines produced by Th9 or Th17 cells. However, the cytokine neutralization approaches clearly highlight the metastasis-promoting role of the signature cytokines of Th9 and Th17 cells. Alongside with the detection of the receptors for IL-9 and IL-17 on the tumor cells, these findings suggest that Th9/IL9 and
Th17/IL17 target both tumor cells and microenvironmental cells. A study using $I l 9 r /$ by Hoelzinger et al. found that IL-9-deficient mice generate tumor-specific $\mathrm{CD} 8^{+} \mathrm{T}$ cells with an enhanced IFN- $\gamma$ secretion and cytotoxic profile when compared with their WT counterparts, which results in early rejection of tumors in breast and colon cancer models. Additionally, neutralization of IL-9 mimicked results obtained in IL-9-deficient mice, with delayed tumor growth in early stages of cancer development (37).

Most importantly, with respect to the translational significance of our findings, targeted inhibition of IL-9 and IL-17 cytokines using neutralizing antibodies was found to decrease EMT and significantly inhibit lung cancer progression and metastasis. This finding (a) corroborates the preceding in vitro studies and the in vivo studies with lung cancer cell and Th9 and/or Th17 coinjection and (b) offers anti-IL-9 and anti-IL-17 treatments as potentially novel immunomodulatory therapeutic concept in lung cancer. This suggestion is in line with previous observations in other cancer types, where IL-17 was noted to induce a tumor-promoting microenvironment, further supporting the view that antiIL-17 intervention might possess therapeutic potential $(38,39)$. Further studies should address the question of how interference with IL- 9 and IL-17 signaling, as suggested by the current findings, might combine with anti-PD1/PD-L1 treatment of lung cancer; how the lung cancer microenvironmental accumulation of IL-9and IL-17-producing $\mathrm{CD} 4^{+} \mathrm{T}$ cells changes in response to these immune checkpoint therapies; and how anti-IL-9/anti-IL-17 strategies effect further important lung cancer microenvironmental cells, such as tumor-associated macrophages.

\section{Methods}

Cell culture. Human adenocarcinoma (A549) and mouse Lewis lung carcinoma cells (LLC1) were purchased from ATCC and maintained in DMEM and RPMI-1640, respectively, and supplemented with $10 \%$ FCS and $1 \%$ penicillin/streptomycin (1\% P/S) (Invitrogen). LLC1 and A549 cells were treated either with CM or with cytokines (IL-9 or IL-17A or IL-17F) in the following concentrations: 1 and $10 \mathrm{ng} / \mathrm{mL}$ (all from R\&D Systems).

Primary cancer cell culture. The University of Giessen Biobank provided cancer cells isolated from human lung tumors (adenocarcinoma, $n=2$ ). Cells were grown in DMEM F12 (supplemented with sodium selenite, ethanolamine, phosphoryl ethanolamine, sodium pyruvate, adenine, and HEPES) and maintained for a maximum of 7-8 passages. Cytokine stimulation and other functional assays were performed similarly as with cell lines described above.

Western blot analysis. After stimulation with cytokines or lymphocyte CM, cells were washed with PBS and then lysed with RIPA lysis buffer for 30 minutes at $4^{\circ} \mathrm{C}$. After protein isolation, immunoblotting was performed using the following primary antibodies: E-cadherin (catalog 3195, Cell Signaling), Vimentin (catalog 5741, Cell Signaling), N-cadherin (catalog 14215, Cell signaling), ZO2 (catalog 2847, Cell Signaling), Cytokeratin 18 (ab668, Abcam), Fibronectin (ab2413, Abcam), and $\beta$-Actin (A2228, MilliporeSigma).

Murine $C D 4^{+} \mathrm{T}$ cell purification and in vitro differentiation. $\mathrm{CD} 4^{+} \mathrm{T}$ cells were purified by negative magnetic cell sorting (CD4 T cell isolation kit, 130-104-454, Miltenyi) from the spleen and lymph nodes of 8- to 12 -week-old mice. For generating Th9 and Th17 subtypes, negatively purified $\mathrm{CD} 4^{+} \mathrm{T}$ cells were further sorted on a FACS AriaI III (BD 
A

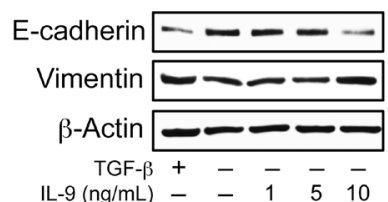

$\mathrm{L}-9(\mathrm{ng} / \mathrm{mL})-\quad$ - 1510
B

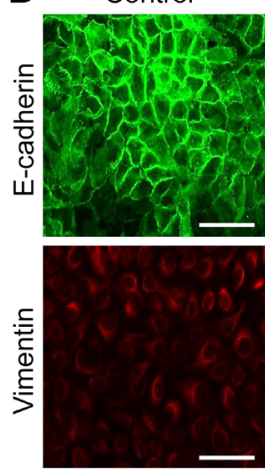

E
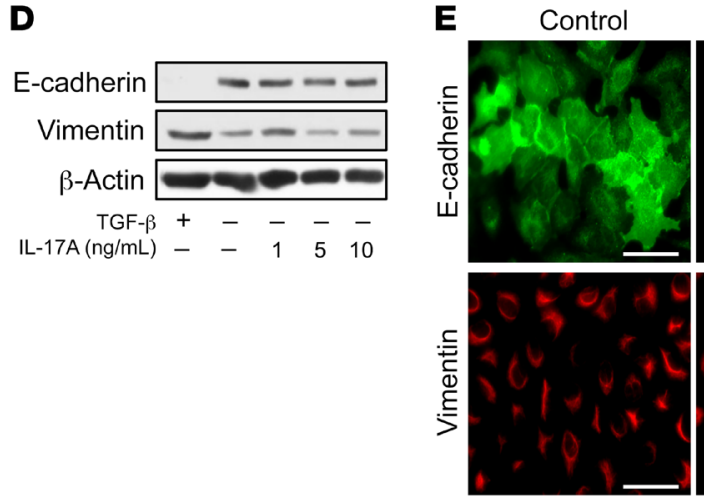

G

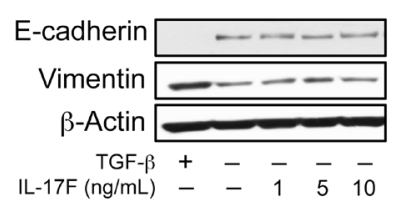

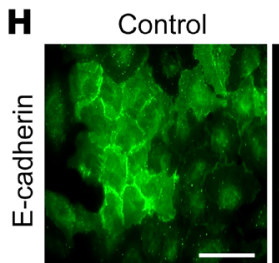
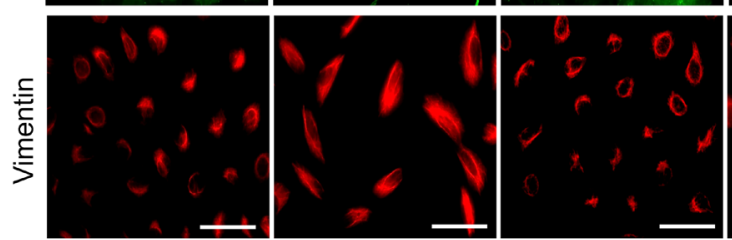

TGF- $\beta$
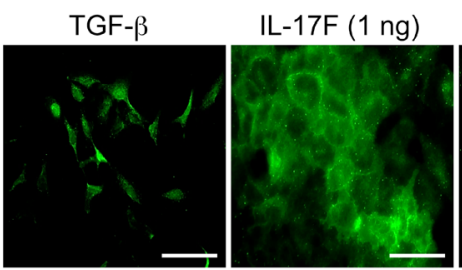
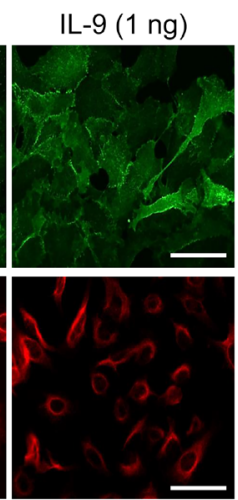

TGF- $\beta$

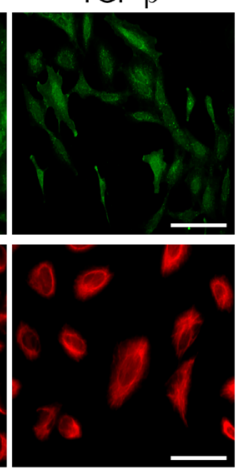

IL-17A (1 ng)
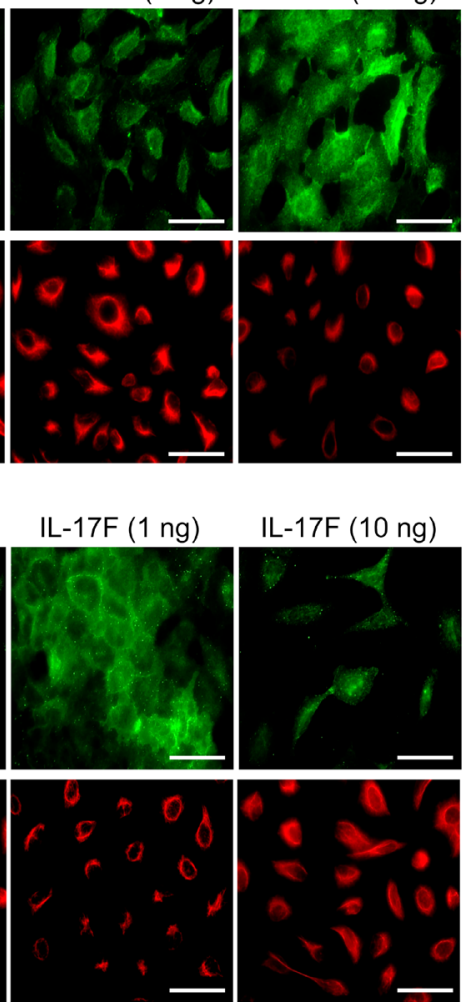

IL-9 (10 ng)
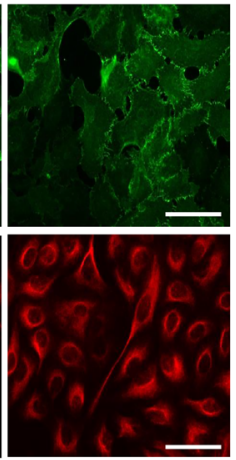

IL-17A (10 ng)
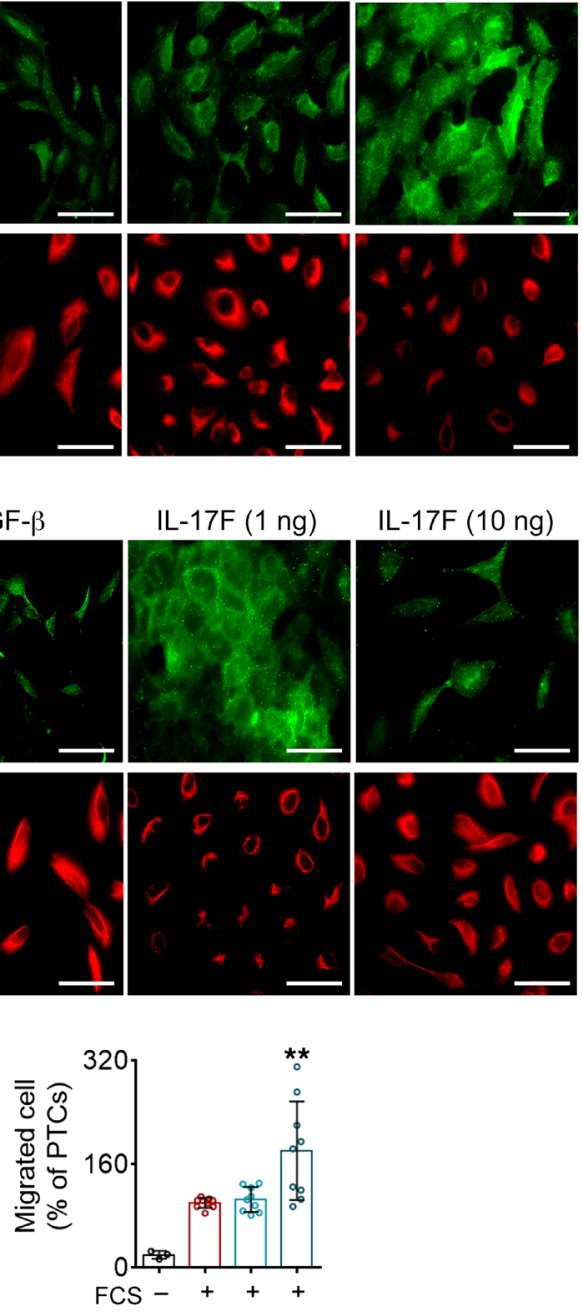

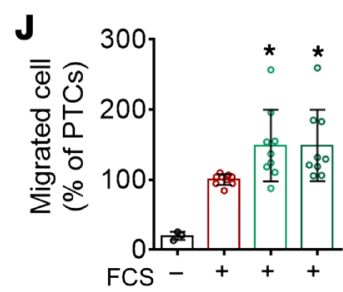

IL-9 (ng/mL)

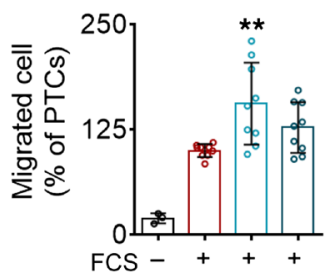

$\mathrm{IL}-17 \mathrm{~A}(\mathrm{ng} / \mathrm{mL})-\quad$ - 110

IL-17F (ng/mL) -

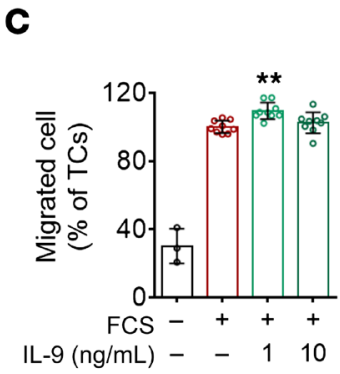

$\mathbf{F}$

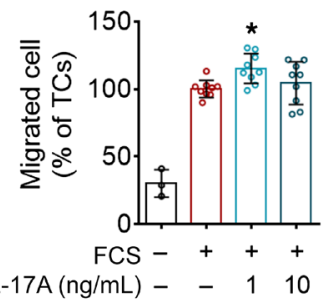

Figure 6. Stimulation with IL-9 and IL-17 results in an EMT-like phenotype in human tumor cells. (A-I) A549 were stimulated with (A-C) IL-9, (D-F) IL-17A, or (G-I) IL-17F. After 48-hour stimulation, (A, D, and G) Western blot analysis of EMT markers (E-cadherin, vimentin) and (B, E, and $\mathbf{H})$ immunocytochemistry of E-cadherin (green) and vimentin (red) were performed. Scale bars: $50 \mu \mathrm{m}$. In addition, after 6-hour stimulation, (C, F, and I) migration assays were performed. (J) Migration of primary human NSCLC cells (PTCs), after 6-hour stimulation with cytokines IL-9, IL-17A, or IL-17F ( $n=9$ replicates). ${ }^{*} P<0.05 ;{ }^{*} P<0.01$ compared with $1 \%$ FCS or basal medium using 1-way ANOVA Dunnett's test.

Biosciences) to obtain naive $\mathrm{CD} 44^{-} \mathrm{CD} 62 \mathrm{~L}^{+} \mathrm{CD} 4^{+} \mathrm{T}$ cells using antiCD4-V450 (RM4-5, BD Biosciences), anti-CD44-PE (IM7, eBioscience), and anti-CD62L-Alexa Fluor 700 (MEL-14, BD Pharmingen) $\mathrm{mAbs}$ in a dilution of 1:500. Sorting purity was maintained typically
$>97 \%$ in postsort analysis. The cells were primed with plate-bound anti-CD3 $\mathrm{mAb}(3 \mu \mathrm{g} / \mathrm{mL}, 145-2 \mathrm{C} 11)$ and anti-CD28 mAb $(5 \mu \mathrm{g} / \mathrm{mL}$, 37.51), both $\mathrm{mAbs}$ produced and purified in-house) in the presence of recombinant human IL-2 (rhIL-2) $(50 \mathrm{U} / \mathrm{mL}$, Novartis), anti-mouse 
IFN- $\gamma(5 \mu \mathrm{g} / \mathrm{mL}, \mathrm{XMG} 1.2$, produced and purified in-house), and antiIL-4 (10\% culture supernatant of clone 11B11). These conditions were considered as neutral conditions (ThO). The cells were also cultured under Th9 conditions by addition of rhTGF- $\beta(1.5 \mathrm{ng} / \mathrm{mL})$ and rmIL-4 $(20 \mathrm{ng} / \mathrm{mL})$ or Th17 conditions by addition of rhTGF- $\beta(1.5 \mathrm{ng} / \mathrm{mL})$ and rmIL-6 $(20 \mathrm{ng} / \mathrm{mL})$. The cells were harvested on day 2 or 3 to obtain differentiated Th9 cells or Th0 and Th17 cells, respectively $(19,20)$. To obtain the CM, differentiated Th0, Th9, and Th17 cells were washed twice with PBS and restimulated with plate-bound anti-CD3 $\mathrm{mAb}(5$ $\mu \mathrm{g} / \mathrm{mL}, 37.51)$ for 24 hours. The $\mathrm{CM}$ were then harvested, centrifuged, filtered, and stored at $80^{\circ} \mathrm{C}$ for further experiments with tumor cells.

Human and mouse microenvironmental cells FACS analysis. For the isolation of mononuclear cells from mouse and human lungs, lungs were dissociated for 45 minutes at $37^{\circ} \mathrm{C}$ by digestion with collagenase $\mathrm{D}$ $(0.5 \mathrm{mg} / \mathrm{mL})$ and DNase I (10 $\mu \mathrm{g} / \mathrm{mL}$; both from Roche) in RPMI medium. Dispersed cells were passed through a $70-\mu \mathrm{m}$ strainer and were pelleted by centrifugation; then they were resuspended, layered onto a Percoll density gradient (GE Healthcare), and centrifuged for 30 minutes at $625 \mathrm{~g}$ and $22^{\circ} \mathrm{C}$. Mononuclear cells were isolated by collection of the interphase fraction between $40 \%$ and $70 \%$ Percoll. After intensive washing in complete RPMI, cells were stained and were analyzed by flow cytometry for CD8a (V500, clone 53-6.7, BD), CD4 (V450, clone RM4-5, BD), B220 (PerCP, clone RA3-6B2, BioLegend), F4/80 (APC, clone BM8, Thermo Fisher). The gating strategy is included in Supplemental Figure 6B. Human lung tumor tissue $(n=5$; adenocarcinoma and squamous cell carcinoma) was obtained from the University of Giessen Biobank and dissociated to obtain a single-cell suspension; then the cells were restimulated for 2.5 hours in the presence of PMA (50 ng/ $\mathrm{mL}$ ), Ionomycin $(1 \mu \mathrm{g} / \mathrm{mL})$, and Brefeldin A $(5 \mu \mathrm{g} / \mathrm{mL})$ (all from MilliporeSigma) for a further 2.5 hours. After the surface staining, the cells were fixed, followed by intracellular staining. The cells were stained with the Zombie NIR fixable viability kit (catalog 423106), anti-CD4 (RPA-T4), anti-CD45RA (HI100), anti-IL-17A (BL168), and anti-IL-9 (MH9A4) (all from BioLegend. Gated CD4 ${ }^{+} \mathrm{CD} 45 \mathrm{RA}$ cells were analyzed for IL-17 or IL-9 on an Aria III using Diva software (v8.0.2.). The gating strategy is included in Supplemental Figure 4.

In vitro coculture model. Lymphocytes were isolated by Ficoll centrifugation gradient from human PBMCs as previously described (40). Briefly, following isolation, lymphocytes were activated with PMA (10 $\mathrm{ng} / \mathrm{mL}$, MilliporeSigma) for 24 hours or by the use of coated plates with anti-CD3/CD28 (16-0037-85, $5 \mu \mathrm{g} / \mathrm{mL}$, and 16-0289-81, $1 \mu \mathrm{g} /$ $\mathrm{mL}$, respectively, Invitrogen). Subsequently, $2.5 \times 10^{6} \mathrm{~A} 549$ cells were seeded with the same amount of PMA or anti-CD3/CD28 activated or nonactivated lymphocytes. A549 and lymphocytes in the presence or absence of PMA or anti-CD3/CD28 were seeded separately as controls. DMEM supplemented with 1\% FCS was used for coculturing. After 24 hours, the supernatant (CM) was harvested, centrifuged, filtered, and stored at $-80^{\circ} \mathrm{C}$ for further experiments. LLC1 cocultures with specific Th subsets Th0, Th9, and/or Th17 were artificially generated as previously described $(19,20)$. LLC1 cells were seeded in a ratio of 1:1 with Th subtypes in RPMI medium supplemented with $2 \%$ FCS. LLC1, Th0, Th9, and Th17 cells were also seeded separately and served as controls. After 24 hours, the CM were harvested, centrifuged, filtered, and stored at $-80^{\circ} \mathrm{C}$ for further experiments.

Proliferation and migration assays. Proliferation assays were performed with serum-starved LLC1, A549, or primary tumor cells exposed for 24 or 48 hours to lymphocyte CM or cytokines (IL-9,
IL-17) using a BrdU colorimetric cell proliferation ELISA (Roche). Chemotactic migration was quantified using a Boyden chamber Transwell assay (8- $\mu \mathrm{m}$ pore size; uncoated filters; BD Biosciences). Either control medium or lymphocyte coculture $\mathrm{CM}$ (in the presence or absence of PMA or anti-CD3/CD28) was provided in the lower chamber, and $4 \times 10^{4}$ lung tumor cells (A549, LLC1, and primary lung tumor cells) were introduced to the upper chamber. Cells were allowed to migrate for 6, 12, and 24 hours. Afterward, migrated cells were fixed, stained, assessed, and quantified with ImageJ software (NIH) $(41,42)$.

ELISA. IL-9, IL-17A, and IL-17F levels were quantified in control, lymphocyte (in the presence or absence of PMA or anti-CD3/CD28), or coculture CM (in the presence or absence of PMA or anti-CD3/ CD28) with the respective Human ELISA Kit from BioLegend per the manufacturer's instructions.

cDNA synthesis and qRT-PCR. Briefly, $1000 \mathrm{ng}$ total RNA was reverse transcribed using the Applied Biosystems High-Capacity cDNA Reverse Transcription Kit (Thermo Fisher Scientific) according the manufacturer's instructions. All real-time RT-PCR reactions were performed on the Stratagene Mx3005P with the MxPro software and the program "SYBR Green with Dissociation curve" to confirm the specificity of each primer. PCR products were separated on a $3 \%$ agarose gel to confirm that every primer yielded 1 specific product. Real-time RT-PCR was performed with the Platinum SYBR Green qPCR SuperMix-UDG (Invitrogen, Life Technologies) and the following conditions: 10 minutes at $95^{\circ} \mathrm{C}$, following 40 cycles of 30 seconds at $95^{\circ} \mathrm{C}, 1$ minute at $58^{\circ} \mathrm{C}-60^{\circ} \mathrm{C}$, and 30 seconds at $72^{\circ} \mathrm{C}$. Analysis was done using MxPro software and GraphPad Prism. All primer pairs were blasted with the NCBI primer blast tool (http://www.ncbi.nlm.nih.gov/tools/primerblast) and purchased from Metabion: E-cadherin, FP, $5^{\prime}$-CCCACCACGTACAAGGGTC-3', and RP, 5'-ATGCCATCGTTGTTCACTGGA-3'; vimentin, FP, 5'-GGAAATGGCTCGTCACCTTCGT-3', and RP, 5'-GCAGAGAAATCCTGCTCTCCT CG-3'; N-cadherin, FP, 5'-TCAGGCGTCTGTAGAGGCTT-3', and RP, 5'-ATGCACATCCTTCGATAAGACTG-3'; ZO1, FP, 5'-ACGGTCCTCTGAGCCTGTAA-3', and RP, 5'-CTGCTTTCTGTTGAGAGGCTGG-3'; ZO2, FP, 5'-GCCCCAGGCATGGAAGAGCTG-3', and RP, 5'-CCCACCCGGGAGCACATCAGA-3'; ZEB1, FP, 5'-AGGATGACCTGCCAACAGACCA-3', and RP, 5'-TCCTTTCTGTCATCCTCCCAGCA-3'; ZEB2, FP, 5'-AGCCTCTGTAGATGGTCCAGAAGAA-3', and RP, 5'-CACTGTACCATTGTTAATTGCGGTC-3'; and cytokeratin 18, FP, 5'-AACAACACCTGCTGTCCGTGT-3', and RP, 5'-TGAAGCTCATGCCCCCAGAAAC-3'.

Mice. We purchased C57BL/6 and Rag1/- animals from The Jackson Laboratory and housed mice under specific pathogen-free condi-

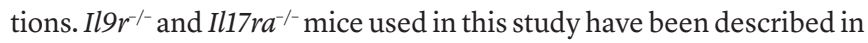
detail previously and were provided in-house $(43,44)$.

Subcutaneous tumor model. Briefly, we coinjected $1 \times 10^{6}$ LLC1 cells with the same amount of Th0, Th9, or Th17 cells subcutaneously into C57BL/6 WT mice. Primary tumor growth of LLC1 cells was monitored as described previously (45).

Metastasis model. We administered LLC1 cells alone or coinjected with Th0, Th9, or Th17 cells at a concentration of $1 \times 10^{6} / 100 \mu \mathrm{L}$ in sterile PBS. C57BL/6, $\mathrm{Ragl}^{-/}, \mathrm{IlOr}^{-/}$, and $\mathrm{Il17ra}^{-/-}$experimental mice $(n=4-6)$ were injected with $100 \mu \mathrm{L}$ cell suspension into the tail vein. Tumor development was monitored for 21 days after injection. Separate lung tissue was harvested from each mouse for nodule count and FACS analysis. Animals treated with antibodies against IL-9 or IL-17 were only injected with LLC1 cells and treated with IL-9 antibody 
A
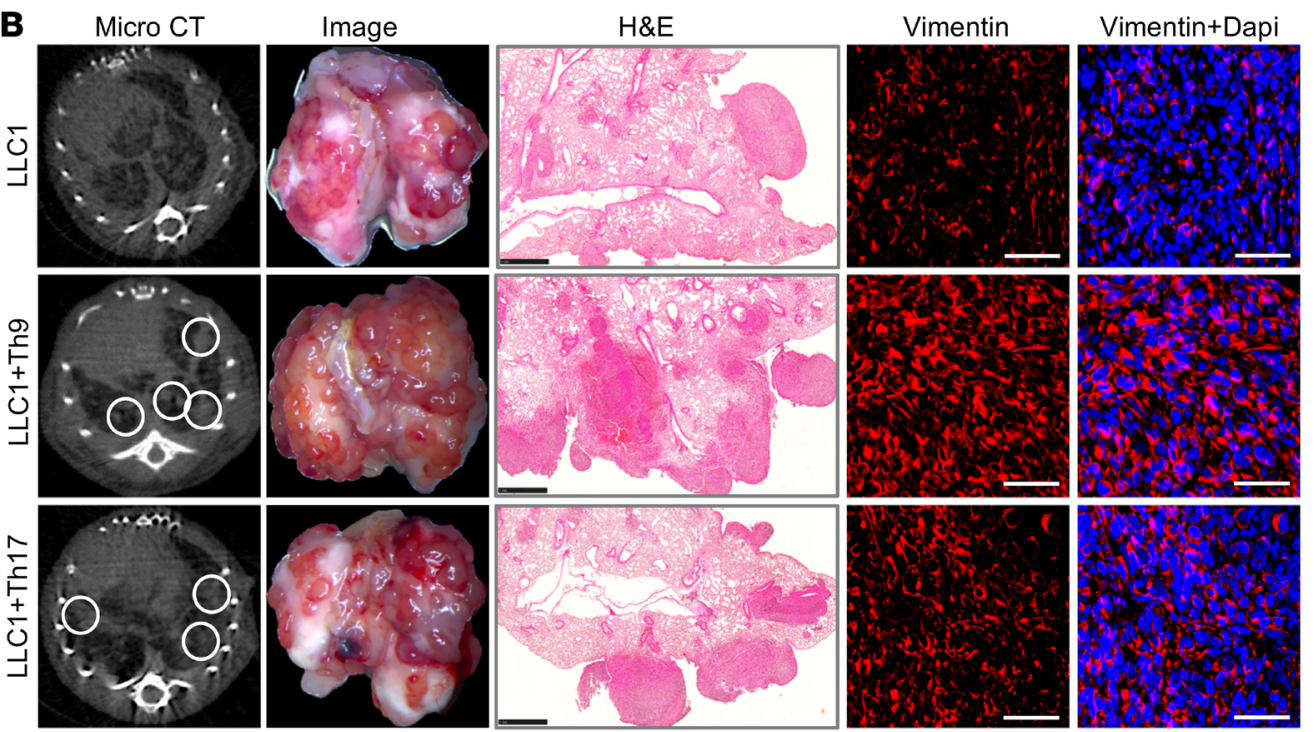

co-injection (i.v.)
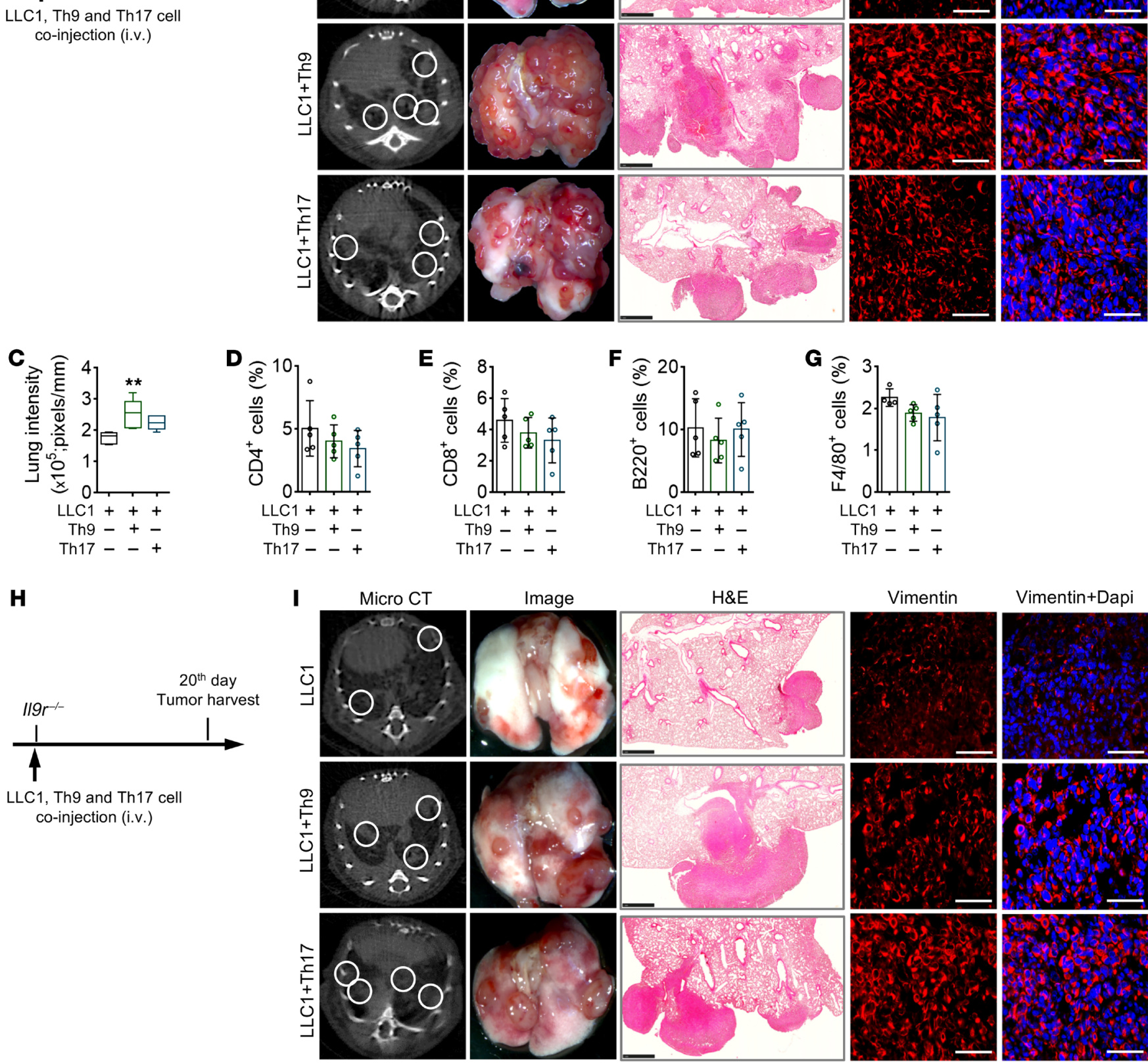

LLC1, Th9 and Th17 cell co-injection (i.v.)
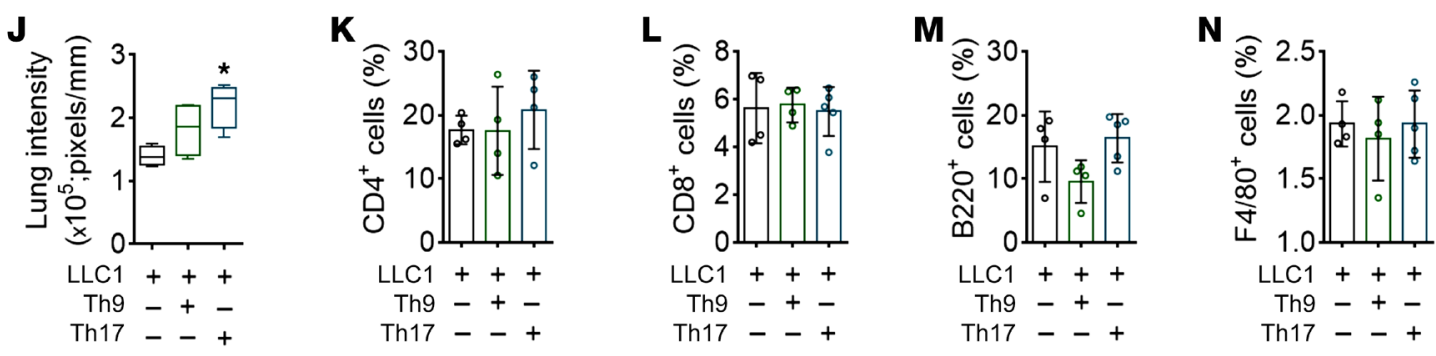
Figure 7. Coinjection of LLC1 cells with Th9 and/or Th17 in II9r or II17 ra mice causes increased metastasis and EMT in the discordant ligandreceptor constellation (Th9 in $/ 117 \mathrm{ra}^{-/-}$mice and Th17 in $/ 19 r^{-1}$ mice), with only limited efficacy in the concordant constellation. Naive CD44-CD62 $\mathrm{L}^{+}$ $\mathrm{CD} 4^{+} \mathrm{T}$ cells were isolated from $\mathrm{C} 57 \mathrm{BL} / 6$ mice and treated with specific cytokines for 2-3 days in order to differentiate into Th9 (TCF- $\beta$ and IL-4), Th17 (TCF- $\beta$ and IL-6) or Th0 (without cytokines) cells. Differentiation was confirmed by performing analysis for IL-9, IL-17, IL-2, and IFN- $\gamma$ production by flow cytometry. Afterward, Th0, Th9 or Th17 cells were coinjected with LLC1 cells into /117 $\mathrm{ra}^{-/-}$(A-E) and $/ 19 \mathrm{r}^{-/-}$(F-J) mice intravenously. (A) Schematic of experimental design. (B) Representative images of micro-CT scans, extracted lung images, H\&E-stained sections, and immunofluorescence staining for vimentin (red) and DAPI (blue) in tumor sections. Scale bars: $50 \mu \mathrm{m}$. (C) Quantification of average lung intensity $(n=5)$. (D-C) FACS analysis of immune cells in lungs from $1 / 17 \mathrm{ra}^{-/-}$mice after coinjection $(n=4)$. (H) Schematic of experimental design. (I) Representative images of micro-CT scans, extracted lungs, H\&E-stained sections and immunofluorescence staining for vimentin (red) and DAPI (blue) in tumor sections. Scale bars: $50 \mu \mathrm{m}$. (J) Quantification of average lung intensity $(n=5)$. (K-N) FACS analysis of immune cells in lungs from $1 / 9 r^{-1-}$ mice after coinjection $(n=4) .{ }^{*} P<0.05 ;{ }^{* *} P<0.01$ compared with LLC1 group using 1-way ANOVA Dunnett's test.

(anti-IL-9; $30 \mu \mathrm{g} /$ mouse clone, MM9C1, provided in-house), IL-17 antibody (anti-IL-17; $100 \mu \mathrm{g} / \mathrm{mouse}$; clone, MAB421, R\&D Systems), or placebo (PBS) administered 4 days after cancer cell injection and followed by continuous intraperitoneal injections on every fourth day. Lungs were harvested on day 21 after injection.

Micro-CT imaging. We imaged mice under anesthesia with isofluorane $2 \%(\mathrm{vol} / \mathrm{vol})$ in $\mathrm{O}_{2}$. Acquisition of images was done with continuous rotation in a high-resolution Micro-CT (SkyScan 1172, Bruker), obtaining 512 projection measurements into a $1024 \times 024$ array (pixel size $0.10 \mathrm{~mm}$ ). Mice scanned for 120 seconds yielded a whole-body exposure of approximately $200 \neq 300 \mathrm{mGy}$ per scan. Reconstruction of images was done with image reconstruction software (NRecon, v1.6, Micro Photonics), and tumor burden is presented as average lung intensity calculated with Image (NIH) through manual segmentation of metastatic tumors as previously described (46).

Opal staining. Lung tumor samples from 66 patients in different cancer stages were obtained. TMAs were constructed from paraffin blocks of selected lung specimens. For each patient, 1.0-mm cores from representative areas of the tumor were taken. Clinical information was obtained from Universitaetsklinikum Muenchen. First, TMA blocks were cut into 5 - $\mu \mathrm{m}$ sections and later deparaffinized, and tissues were fixed in $10 \%$ neutral buffered formalin before antigen retrieval in heated AR6 buffer (pH 6.0) for 15 minutes (EZ Retriever microwave, BioGenex). Each section was put through 7 sequential rounds of staining, each including a protein block with $5 \%$ BSA, followed by primary antibody incubation overnight (Cytokeratin, 1:500, DAKO, MA126237; PU.1, 1:200, Abcam, ab76543; IL-9, 1:200, Abcam, ab181397; STAT3, 1:200, Cell Signaling, 12640S; IL-17, 1:200, Abcam, ab79056; and CD4, 1:100, Abcam, ab133616) and corresponding secondary horseradish peroxidase-conjugated polymer and Opal fluorophores $(18,47)$. High versus low ratio of Th9/CD4 and Th17/CD4 was defined through calculation of median intensity.

RNA-Seq and bioinformatical analysis. RNA was isolated from LLC1 cells after sorting using the miRNeasy micro Kit (Qiagen) combined with on-column DNase digestion (DNase-Free DNase Set, Qiagen) to avoid genomic DNA contamination. RNA and library prepa- ration integrity were verified with a BioAnalyzer 2100 (Agilent) or LabChip Gx Touch 24 (PerkinElmer). One $\mu$ g total RNA was used as input for SMARTer Stranded Total RNA Sample Prep Kit, HI Mammalian (Clontech). Sequencing was performed on the NextSeq500 instrument (Illumina) using v2 chemistry, resulting in average of 30 $\mathrm{M}$ reads per library, with $1 \times 75$-bp single-end setup. The resulting raw reads were assessed for quality, adapter content, and duplication rates with FastQC (48). Reaper version 13-100 was used to trim reads after a quality drop below a mean of $\mathrm{Q} 20$ in a window of 10 nucleotides. Reads between 30 and 150 nucleotides only were cleared for further analysis. Trimmed and filtered reads were aligned versus the Ensembl mouse genome version mm10 (GRCm38) using STAR 2.4.0a with the parameter "--outFilterMismatchNoverLmax 0.1" to increase the maximum ratio of mismatches to mapped length to $10 \%$ (49). The number of reads aligning to genes was counted with featureCounts 1.4.5p1 tool from the Subread package (50). Only reads mapping at least partially inside exons were admitted and aggregated per gene. Reads that are overlapping multiple genes or aligning to multiple regions were excluded. Differentially expressed genes were identified using DESeq2 version 1.62 (51). Only genes with a minimum fold change of \pm 1.5 ( $\log 2 \pm 0.59$ ), a maximum Benjamini-Hochberg corrected $P$ value of 0.05 , and a minimum combined mean of 5 reads were deemed to be significantly differentially expressed. RNA-Seq data were deposited in the Gene expression omnibus archive (accession GSE145186). The Ensemble annotation was enriched with UniProt data (release 06.06.2014) based on Ensembl gene identifiers (activities at the Universal Protein Resource).

Immunostaining. Immunocytochemistry was performed on A549 cells as previously described with the following primary antibodies: E-cadherin and vimentin (both diluted 1:200 Cell Signaling, clone D21H3). For IHC staining, 3- $\mu \mathrm{m}$ paraffin lung tissue sections were rehydrated and antigen retrieval was achieved with citrate buffer/ heat treatment. Lung tissue sections were blocked and incubated with primary antibodies against vimentin (1:200 Cell Signaling, clone $\mathrm{D} 21 \mathrm{H} 3$ ) or subjected to H\&E staining for metastatic burden. Indirect immunofluorescence was conducted by incubation with Alexa Fluor 488- or Alexa Fluor 594-conjugated secondary antibodies (A11008 and A27039, respectively, Invitrogen), and nuclei were counterstained with DAPI. All fluorescence images were acquired with the same exposure time at $\times 40$ magnification.

Statistics. Statistical analysis was performed with GraphPad Prism 7 software. One-way ANOVA followed by Dunnett's comparison test was used to compare the means of individual groups to the control group. For other analyses, we used 2-way ANOVA. Two independent groups were compared with 2-tailed Student's $t$ test. Data are expressed as mean \pm SEM.

Study approval. All experiments were performed in accordance with the Animal Welfare Act and were approved by the local authorities under reference V54-19c20/15-B2/1062 (Regierungspräsidium Darmstadt, Hessen, Germany). Written informed consent was obtained from patients for tissue sample collection, according to the research proposal approved by the Institutional Review Board at the Medical Faculty of the University of Giessen (reference AZ 58/15).

\section{Author contributions}

RS, SSP, YS, MH, and WS conceived and designed the study. YS, $\mathrm{XZ}, \mathrm{DB}, \mathrm{AS}, \mathrm{HR}, \mathrm{A}$. Weigert, FP, YZ, BB, SG, and FG acquired, 
A a-IL-9/IL-17 treatment

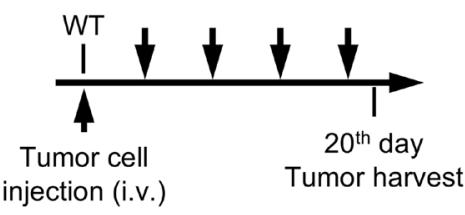

C

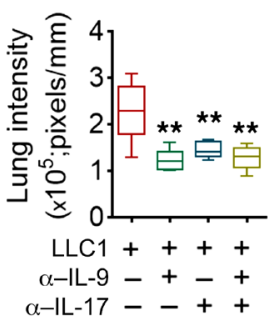

D

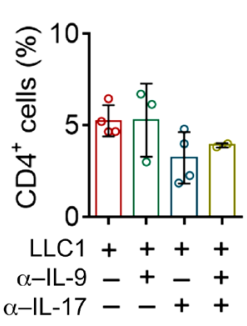

E

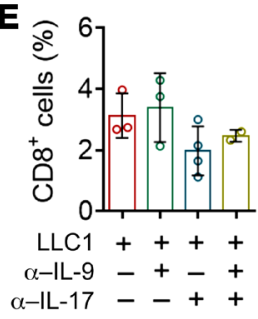

B
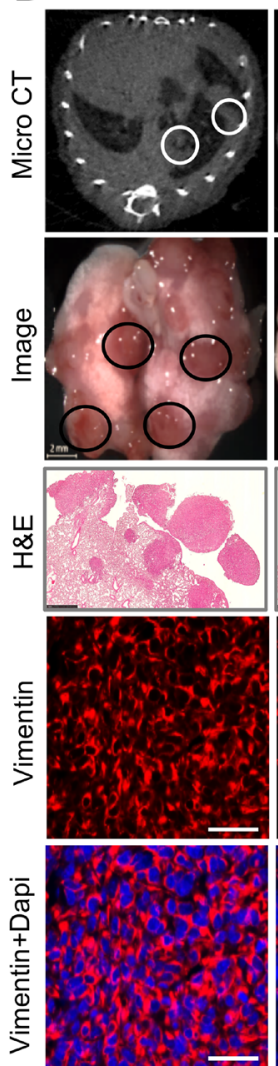

LLC1+ $+\alpha-I L-9$
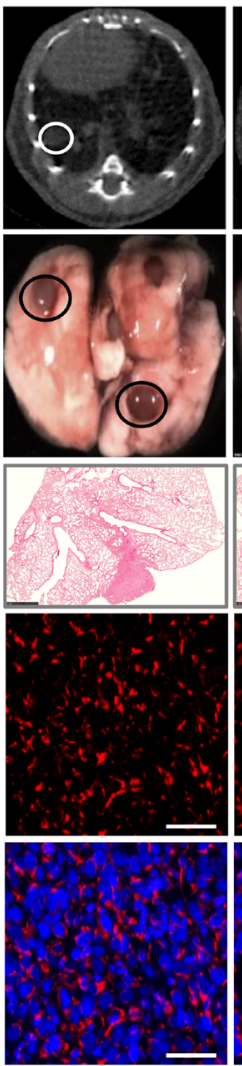

LLC1+
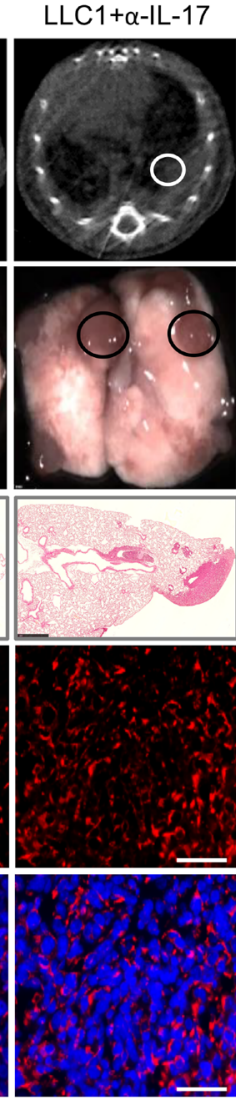

$\alpha-I L-9+\alpha-I L-17$
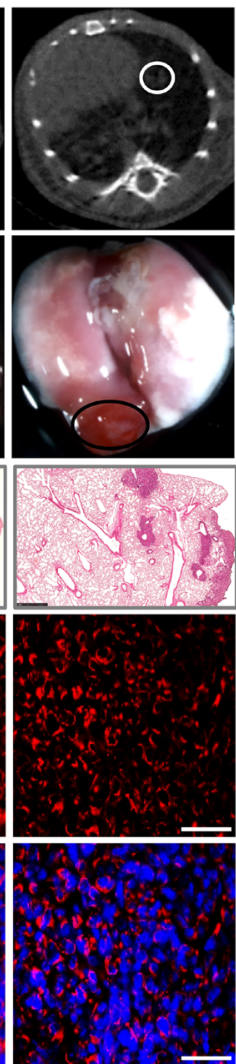

$\mathbf{F}$
${ }^{\mathrm{rn} 9}$

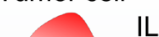

Th17

Tumor cell
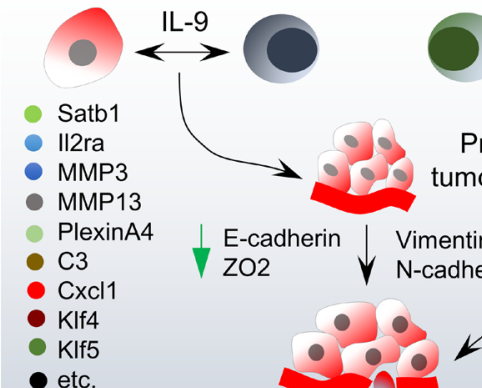

IL-17 7 $\triangle \mathrm{Myc}$ A Pola2

$\triangle$ Alg2

A Scrn2

$\Delta$ Col5a1

Tcf12

$\triangle$ Atf2

$\triangle \mathrm{Cdk} 4$

$\triangle$ Kllf7

- etc.

$\Delta$ etc.

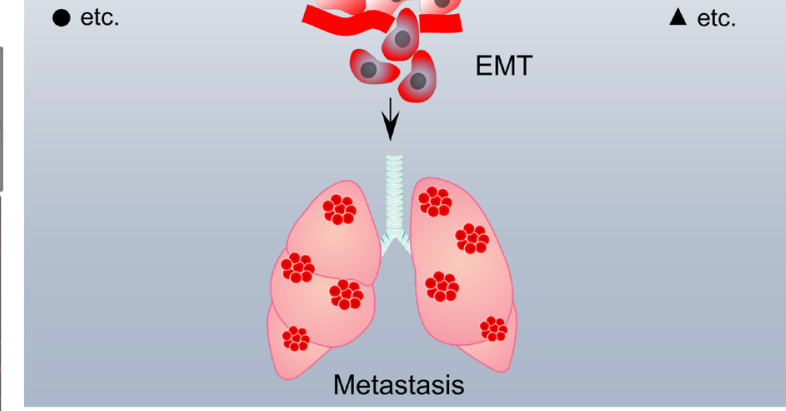

Figure 8. Neutralization of IL-9 (anti-IL-9) and IL-17 (anti-IL-17) reduces tumor metastasis to the lung. (A) Schematic of experimental design. (B) Representative pictures of micro-CT scans, extracted lung images, H\&E-stained sections, and immunofluorescence of vimentin (red) in sections of lungs with metastatic nodules after treatment with neutralizing antibodies on day 20 after intravenous injection of LLC1 cells. Scale bars: $50 \mu \mathrm{m}$. (C) Quantification of average lung intensity in treated mice $(n=5)$. ( $(\mathbf{D}$ and $\mathbf{E})$ FACS analysis of $C D 4^{+}$and CD8 ${ }^{+}$immune cells in lungs from treated mice after coinjection $(n=3-4)$. ${ }^{*} P<0.01$, compared with nontreated mice using 1-way ANOVA Dunnett's test. (F) Summary with proposed players in the prometastatic and EMT-inducing effect of Th9 and Th17 cells in lung cancer. The specific subsets through secretion of their signature cytokines, IL-9 and IL-17, induce the transition of tumor cells to a highly migratory mesenchymal phenotype characterized by loss of epithelial markers (E-cadherin, ZO2) and gain of mesenchymal markers (vimentin, N-cadherin). This effect is associated with corresponding EMT- and migration-related protein and transcription factor gene regulation in tumor cells that are in direct crosstalk with a specific Th subtype.

analyzed, and interpreted data. HW, AT and LF provided human samples. BW, LD, JCR, and A. Waisman provided $I l 9 \mathrm{r}^{-/}$and $\mathrm{Ill} \mathrm{ra}^{-/-}$mice. TB provided IL-9 antibody. RS, SSP, MH, YS, and WS prepared the manuscript.

\section{Acknowledgments}

We thank Andrea Mohr, Yanina Knepper, Vanessa Golchert, and Marianne Hoeck for excellent technical assistance and Astrid Wietelmann for great support in micro-CT imaging. This work was supported by the Max Planck Society; Verein zur Förderung der Krebsforschung in Gießen e.V.; a Von-Behring-Röntgen-Stiftung grant (62-0013); a Rhön Klinikum AG grant; the German Center for Lung Research (DZL); the Cardio-Pulmonary Institute (CPI); DFG grants SFB 1213 (projects A01 and A05) and SFB1292; DFG HU 1824/5-1, HU 1824/ 7-1, and HU 1824/9-1; Fresenius Stiftung 2015_A232; and the European Research Council (ERC) Consolidator Grant (\#866051).

Address correspondence to: Rajkumar Savai, Molecular Mechanisms in Lung Cancer, Department of Lung Development and Remodelling, Max Planck Institute for Heart and Lung Research, Parkstrasse 1, D-61231 Bad Nauheim, Germany. Phone: 49.6032.705.420; Email: rajkumar.savai@mpi-bn.mpg.de. 
1. Torre LA, Siegel RL, Jemal A. Lung cancer statistics. Adv Exp Med Biol. 2016;893:1-19.

2. Hensing T, Chawla A, Batra R, Salgia R. A personalized treatment for lung cancer: molecular pathways, targeted therapies, and genomic characterization. Adv Exp Med Biol. 2014;799:85-117.

3. Shalapour S, Karin M. Immunity, inflammation, and cancer: an eternal fight between good and evil. J Clin Invest. 2015;125(9):3347-3355.

4. Mittrücker HW, Visekruna A, Huber M. Heterogeneity in the differentiation and function of $\mathrm{CD} 8^{+} \mathrm{T}$ cells. Arch Immunol Ther Exp (Warsz). 2014;62(6):449-458.

5. Hadrup S, Donia M, Thor Straten P. Effector CD4 and CD8 $T$ cells and their role in the tumor microenvironment. Cancer Microenviron. 2013;6(2):123-133.

6. Hung K, Hayashi R, Lafond-Walker A, Lowenstein C, Pardoll D, Levitsky H. The central role of CD4(+) T cells in the antitumor immune response. J Exp Med. 1998;188(12):2357-2368.

7. Kim HJ, Cantor H. CD4 T-cell subsets and tumor immunity: the helpful and the not-so-helpful. Cancer Immunol Res. 2014;2(2):91-98.

8. Pellegrini P, Berghella AM, Del Beato T, Cicia S, Adorno D, Casciani CU. Disregulation in TH1 and $\mathrm{TH} 2$ subsets of $\mathrm{CD} 4+\mathrm{T}$ cells in peripheral blood of colorectal cancer patients and involvement in cancer establishment and progression. Cancer Immunol Immunother. 1996;42(1):1-8.

9. Nishimura $\mathrm{T}$, et al. Distinct role of antigenspecific T helper type 1 (Th1) and Th2 cells in tumor eradication in vivo. J Exp Med. 1999;190(5):617-627.

10. Purwar R, et al. Robust tumor immunity to melanoma mediated by interleukin-9-producing $\mathrm{T}$ cells. Nat Med. 2012;18(8):1248-1253.

11. Lv X, Feng L, Ge X, Lu K, Wang X. Interleukin-9 promotes cell survival and drug resistance in diffuse large B-cell lymphoma. J Exp Clin Cancer Res. 2016;35(1):106

12. Ye ZJ, et al. Differentiation and immune regulation of IL-9-producing CD4+ T cells in malignant pleural effusion. Am J Respir Crit Care Med. 2012;186(11):1168-1179.

13. Muranski P, et al. Tumor-specific Th17-polarized cells eradicate large established melanoma. Blood. 2008;112(2):362-373.

14. Grivennikov SI, et al. Adenoma-linked barrier defects and microbial products drive IL-23/ IL-17-mediated tumour growth. Nature. 2012;491(7423):254-258.

15. Hus I, et al. Th17/IL-17A might play a protective role in chronic lymphocytic leukemia immunity. PLOS ONE. 2013;8(11):e78091.

16. Duan MC, et al. Disturbed Th17/Treg balance in patients with non-small cell lung cancer. Inflammation. 2015;38(6):2156-2165.

17. Ricciardi M, et al. Epithelial-to-mesenchymal transition (EMT) induced by inflammatory priming elicits mesenchymal stromal cell-like immune-modulatory properties in cancer cells. Br J Cancer. 2015;112(6):1067-1075.

18. Carstens JL, et al. Spatial computation of intratumoral $\mathrm{T}$ cells correlates with survival of patients with pancreatic cancer. Nat Commun.
2017;8:15095.

19. Campos Carrascosa L, et al. Reciprocal regulation of the Il9 locus by counteracting activities of transcription factors IRF1 and IRF4. Nat Commun. 2017;8:15366.

20. Huber M, et al. IL-17A secretion by CD8+ T cells supports Th17-mediated autoimmune encephalomyelitis. J Clin Invest. 2013;123(1):247-260.

21. Prudkin L, et al. Epithelial-to-mesenchymal transition in the development and progression of adenocarcinoma and squamous cell carcinoma of the lung. Mod Pathol. 2009;22(5):668-678.

22. Chen X, et al. Increased IL-17-producing cells correlate with poor survival and lymphangiogenesis in NSCLC patients. Lung Cancer. 2010;69(3):348-354.

23. Abousamra NK, Salah El-Din M, Helal R. Prognostic value of Th17 cells in acute leukemia. Med Oncol. 2013;30(4):732.

24. Cunha LL, et al. Infiltration of a mixture of immune cells may be related to good prognosis in patients with differentiated thyroid carcinoma. Clin Endocrinol (Oxf). 2012;77(6):918-925.

25. Tan H, Wang S, Zhao L. A tumour-promoting role of Th9 cells in hepatocellular carcinoma through CCL2O and STAT3 pathways. Clin Exp Pharmacol Physiol. 2017;44(2):213-221.

26. Radisky DC, et al. Rac1b and reactive oxygen species mediate MMP-3-induced EMT and genomic instability. Nature. 2005;436(7047):123-127.

27. Zigrino P, et al. Stromal expression of MMP-13 is required for melanoma invasion and metastasis. J Invest Dermatol. 2009;129(11):2686-2693.

28. Cho MS, et al. Complement component 3 is regulated by TWIST1 and mediates epithelial-mesenchymal transition. J Immunol. 2016;196(3):1412-1418.

29. Kigel B, Rabinowicz N, Varshavsky A, Kessler O, Neufeld G. Plexin-A4 promotes tumor progression and tumor angiogenesis by enhancement of VEGF and bFGF signaling. Blood. 2011;118(15):4285-4296.

30. Lu J, et al. 14-3-3zeta Cooperates with ErbB2 to promote ductal carcinoma in situ progression to invasive breast cancer by inducing epithelialmesenchymal transition. Cancer Cell. 2009;16(3):195-207.

31. Nishimura Y, et al. Overexpression of YWHAZ relates to tumor cell proliferation and malignant outcome of gastric carcinoma. $\mathrm{Br} \mathrm{JCancer}$. 2013;108(6):1324-1331.

32. Fan T, et al. Up-regulation of 14-3-3zeta in lung cancer and its implication as prognostic and therapeutic target. Cancer Res. 2007;67(16):7901-7906.

33. Chen $\mathrm{CH}$, Chuang SM, Yang MF, Liao JW, Yu SL, Chen JJ. A novel function of YWHAZ/ $\beta$-catenin axis in promoting epithelial-mesenchymal transition and lung cancer metastasis. Mol Cancer Res. 2012;10(10):1319-1331.

34. Ding X, Wang X, Gong Y, Ruan H, Sun Y, Yu Y. KLF7 overexpression in human oral squamous cell carcinoma promotes migration and epithelial-mesenchymal transition. Oncol Lett. 2017;13(4):2281-2289.

35. Gao Y, et al. Beyond proliferation: KLF5 promotes angiogenesis of bladder cancer through directly regulating VEGFA transcription. Oncotarget. 2015;6(41):43791-43805.

36. Mehner C, Hockla A, Miller E, Ran S, Radisky DC, Radisky ES. Tumor cell-produced matrix metalloproteinase 9 (MMP-9) drives malignant progression and metastasis of basal-like triple negative breast cancer. Oncotarget. 2014;5(9):2736-2749.

37. Hoelzinger DB, Dominguez AL, Cohen PA, Gendler SJ. Inhibition of adaptive immunity by IL9 can be disrupted to achieve rapid T-cell sensitization and rejection of progressive tumor challenges. Cancer Res. 2014;74(23):6845-6855.

38. He D, et al. IL-17 promotes tumor development through the induction of tumor promoting microenvironments at tumor sites and myeloid-derived suppressor cells. JImmunol. 2010;184(5):2281-2288.

39. Benevides L, et al. IL17 promotes mammary tumor progression by changing the behavior of tumor cells and eliciting tumorigenic neutrophils recruitment. Cancer Res. 2015;75(18):3788-3799.

40. Bøyum A. Isolation of lymphocytes, granulocytes and macrophages. Scand J Immunol. 1976;5(Suppl 5):9-15.

41. Pullamsetti SS, et al. Lung cancer-associated pulmonary hypertension: Role of microenvironmental inflammation based on tumor cell-immune cell cross-talk. Sci Transl Med. 2017;9(416):eaai9048.

42. Savai R, et al. Pro-proliferative and inflammatory signaling converge on FoxO1 transcription factor in pulmonary hypertension. Nat Med. 2014;20(11):1289-1300.

43. Steenwinckel V, et al. IL-13 mediates in vivo IL-9 activities on lung epithelial cells but not on hematopoietic cells. JImmunol. 2007;178(5):3244-3251.

44. El Malki K, et al. An alternative pathway of imiquimod-induced psoriasis-like skin inflammation in the absence of interleukin-17 receptor a signaling. J Invest Dermatol. 2013;133(2):441-451.

45. Schmall A, et al. Macrophage and cancer cell cross-talk via CCR2 and CX3CR1 is a fundamental mechanism driving lung cancer. Am J Respir Crit Care Med. 2015;191(4):437-447.

46. Krupnick AS, et al. Quantitative monitoring of mouse lung tumors by magnetic resonance imaging. Nat Protoc. 2012;7(1):128-142.

47. Mezheyeuski A, et al. Multispectral imaging for quantitative and compartment-specific immune infiltrates reveals distinct immune profiles that classify lung cancer patients. JPathol. 2018;244(4):421-431.

48. Andrews S. 2010, FastQC: a quality control tool for high throughput sequence data. http://www. bioinformatics.babraham.ac.uk/projects/fastqc. Accessed May 9, 2020.

49. Dobin A, et al. STAR: ultrafast universal RNA-seq aligner. Bioinformatics. 2013;29(1):15-21.

50. Liao Y, Smyth GK, Shi W. featureCounts: an efficient general purpose program for assigning sequence reads to genomic features. Bioinformatics. 2014;30(7):923-930.

51. Love MI, Huber W, Anders S. Moderated estimation of fold change and dispersion for RNA-seq data with DESeq2. Genome Biol. 2014;15(12):550. 